\title{
« Principe 7 » De La Pédagogie, Effet Zeeman-Effet Prérequis, Architecture Pyramidale Du Système Lmd: Mise En Guvre D'une Pédagogie Discriminatoire Pour Un Enseignement De Qualité
}

\author{
Ibrahima Sakho \\ Département de Physique, UFR Sciences et Technologies \\ Université Assane Seck de Ziguinchor, Ziguinchor, Sénégal
}

Doi: 10.19044/esj.2018.v14n24p159 URL:http://dx.doi.org/10.19044/esj.2018.v14n24p159

\begin{abstract}
In this work, we demonstrate by analogy with the quantified levels of atomic systems, that the levels of education from elementary to Superior are "quantified". The transition of a learner from one level of instruction to another is analogous to an electronic transition between atomic levels. The notions of ground state, excited states and ionized state of atomic systems have their equivalents in the school and university systems. It is demonstrated in this work that the number 7 is revealed in all countable elements of an educational system. This leads to the statement of the "principle 7 " of pedagogy. Thus, there are 7 levels of instruction or teaching-learning in the elementary, 7 levels at the middle-level and 7 levels of teaching-learning at the higher level. In addition, there are 7 degrees, 7 hours of work of the learner in the school space. The application of "Principle 7 of pedagogy" to the determination of the number of classes per cycle and the number of students per classroom is discussed. Moreover, it is shown in this work, that the Pauli Exclusion Principle allows to account for the correct occupation of the tablesbenches by the learners and that and the indiscernibility principle of the particles accounts for the necessity of the wearing school clothes. By analogy with the Zeeman Effect, it is shown that all levels of teaching-learning are degenerate. The lifting of degeneration by "Prerequisite Effect" highlights the need to put into practice a discriminatory pedagogy for quality education at the elementary level as well as at the higher level. Finally, it is demonstrated by analogy with the tightening of the atomic levels with the increase of the principal quantum number, that the architecture of the LDM must have the appearance of a pyramid, the base being constituted by the level License and the summit by the $\mathrm{PhD}$ level. Moreover, the increase in the number of 180/120/180 credits does not follow the pyramidal architecture of the LMD and that it should mathematically decrease according to the ratio $\mathrm{L} / \mathrm{M}=\mathrm{M}$ /
\end{abstract}


$\mathrm{D}=3 / 2$; which corresponds to the $180 / 120 / 80$ pyramidal progression. If a credit corresponds to 20 hours of work in $\mathrm{L}$ and $\mathrm{M}$, it should correspond to 45 hours of work in D.

Keywords: Atomic systems, teaching-learning level, learning level, Pauli Exclusion Principle, indiscernibility principle, Zeeman Effect, Prerequisite Effect, discriminatory pedagogy, LMD system.

\section{Résumé}

Dans ce travail, nous démontrons par une analogie avec les niveaux quantifiés des systèmes atomiques, que les niveaux d'enseignement de l'élémentaire au Supérieur sont « quantifiés ». Le passage d'un apprenant d'un niveau d'instruction à un autre est analogue à une transition électronique entre les niveaux atomiques. Les notions d'état fondamental, d'états excités et d'état ionisé des systèmes atomiques ont leurs équivalents dans les systèmes scolaire et universitaire. Il est démontré dans ce travail que le chiffre 7 se révèle dans tous les éléments dénombrables d'un système éducatif. Ce qui conduit à l'énoncé du «principe 7 de la pédagogie». Ainsi, il existe 7 niveaux d'instruction ou d'enseignement-apprentissage dans l'élémentaire, 7 niveaux au moyen-secondaire et 7 niveaux d'enseignement-apprentissage au supérieur. De même, il existe 7 diplômes, 7 heures de travail de l'apprenant dans l'espace scolaire. L'application du « principe 7 » de la pédagogie à la détermination du nombre de classes par cycle et du nombre d'apprenants par salle de classe est discutée. De plus, il est montré dans ce travail, que le principe d'exclusion de Pauli permet de rendre compte de l'occupation correcte des tables-bancs par les apprenants et que et le principe d'indiscernabilité des particules rend compte de la nécessité du port de la tenue scolaire. Par analogie à l'Effet Zeeman, il est démontré que tous les niveaux d'enseignement-apprentissage sont dégénérés. La levée de dégénérescence par "Effet Prérequis » met en évidence la nécessite de mise en pratique d'une pédagogie discriminatoire pour un enseignement de qualité au niveau élémentaire comme au niveau supérieur. Enfin, il est démontré par analogie aux resserrements des niveaux atomiques avec l'accroissement du nombre quantique principal, que l'architecture du LDM doit avoir l'allure d'une pyramide, la base étant constituée par le niveau Licence et le sommet par le niveau Doctorat. De plus, la progression du nombre de crédits 180/120/180 ne suit pas l'architecture pyramidale du LMD et qu'il devrait mathématiquement décroitre selon le rapport $\mathrm{L} / \mathrm{M}=\mathrm{M} / \mathrm{D}=3 / 2$; ce qui correspond à la progression pyramidale 180/120/80. Si un crédit correspond à $20 \mathrm{~h}$ de travail en L et M, il devrait correspondre à $45 \mathrm{~h}$ de travail en $\mathrm{D}$. 
Mots-clés : Systèmes atomiques, niveaux d'enseignement-apprentissage, niveaux d'instruction, principe d'exclusion de Pauli, principe d'indiscernabilité, Effet Zeeman, Effet Prérequis, pédagogie discriminatoire, système LMD.

\section{Introduction}

Historiquement, la pédagogie désignait l'art d'éduquer. Buisson, (1911) qui fut inspecteur général de l'instruction publique définit l'éducation comme l'action exercée par les générations adultes sur celles qui ne sont pas encore mûres pour la vie sociale. L'éducation a pour objectif de susciter et de développer chez l'enfant un certain nombre d'états physiques, intellectuels et mentaux que réclament de lui, et la société politique dans son ensemble et le milieu social auquel il est particulièrement destiné. La pédagogie en tant qu'art d'éduquer était donc dédiée à l'enfant. Aujourd'hui, la pédagogie a une portée plus générale: elle est destinée aussi bien à l'enfant (élève - de 18 ans) qu'à l'adulte (étudiant + de 18 ans). Dans son acception moderne, la pédagogie rassemble les méthodes et pratiques d'enseignement et d'éducation. De plus, elle renvoie à toutes les qualités requises pour transmettre des compétences de savoirs tout court (connaissances), de savoir-faire théorique et / ou pratique et de savoir-être. Mais, les notions d'états physiques, intellectuels et mentaux d'un apprenant sont des états immatériels donc inobservables qui s'apparentent aux états quantiques des systèmes physiques (électrons, atomes, molécules, etc.). En 2011, nous avions montré par analogie aux systèmes atomiques, que l'espace scolaire est «quantifié » (Sakho, 2011). Cette publication résultait d'une compilation des idées que nous avions développées lors d'une conférence animée pour des élèves au lycée de Bambey au Sénégal (Sakho, 2008). De plus, lors d'une conférence animée à l'Université Assane Seck de Ziguinchor (Sakho, 2013), nous montrâmes que l'espace universitaire est aussi «quantifié » et que les niveaux d'instruction sont au nombre de 7 pour chaque cycle. Il en est de même des diplômes qui sont aussi au nombre de 7. De plus, nous annoncions à l'occasion d'une conférence animée à l'Université Alioune Diop de Bambey (Sakho, 2016), qu'il est possible de comprendre tous les comportements de l'homme (sans distinction de sexe) à partir de son espace existentiel qui n'est rien d'autre qu'un espace vectoriel à deux dimensions engendré par les vecteurs de base $\mid$ vie $\rangle$ et $\mid$ mort $\rangle$. Mais des chercheurs du monde entier penchent aussi sur la question. Lors d'une conférence, le Dr Béatrice Milbert expliquait que «la physique quantique appliquée à la biologie et à la médecine offre une opportunité pour appréhender le "corps énergie" et comprendre ainsi les médecines dites énergétiques multiséculaires » (Milbert, 2012). De plus, le Prof Robert Lanza, de l'Université médicale de Wake Forest, en Californie du Nord, explique dans son livre (Lanza, 2010) que «la physique quantique prouve qu'il y a une vie 
après la mort.. ». Si la physique quantique prouve qu'il y a une vie après la mort, elle doit pouvoir expliquer des choses moins complexes telles que les divers comportements de l'homme sur terre. Mais, l'homme fait une seule chose sur terre : il ne fait qu'agir sur son environnement. Or, toutes les activités de l'homme ont lieu périodiquement: chaque semaine ou tous les 7 jours. Nous montrons dans ce travail que le chiffre 7 se révèle être le « chiffre de la pédagogie ». Ce qui permet d'énoncer ce que nous appelons «le principe 7 » de la pédagogie appliqué à la détermination du nombre de niveaux d'enseignement-apprentissage, du nombre d'heures de travail apprenants, du nombre de diplômes, du nombre de salles de classe par cycle et du nombre d'apprenants par classe pour un cycle donné. Le plan adopté est le suivant. Dans la deuxième section, nous déclinons la méthodologie adoptée dans ce travail consistant à profit la démarche analogique à partir d'une situationsource (système atomique) et d'une situation-cible (système pédagogique). Les résultats obtenus sont présentés et discutés dans la troisième section. Les équivalents des notions d'état fondamental, d'états excités et d'état ionisé des systèmes atomiques sont précisés dans le cas des systèmes pédagogiques (systèmes scolaires et universitaires). Nous discuterons aussi dans cette troisième section, de la perte de performance induite par l'instauration du régime de la journée continue. De plus, l'application du «principe 7 » de la pédagogie à la détermination du nombre de classes par cycle et du nombre d'élèves par salle de classe est discutée. Les dualités principe d'exclusion de Pauli-occupation correcte des tables-bancs et principe d'indiscernabilitétenues scolaires des apprenants sont aussi discutées. Sur la base de l'effet Zeeman, nous démontrerons que tous les niveaux d'enseignementapprentissage sont « dégénérés ». La levée de cette dégénérescence par «Effet Prérequis » permet de mettre en pratique une pédagogie discriminatoire pour un enseignement efficace en classe dans les établissements scolaires et universitaires. De plus, dans cette section, nous mettons à profit le resserrement des niveaux atomiques avec l'accroissement du nombre quantique principal, pour montrer que le système LDM doit avoir une architecture pyramidale du point de vue des modules enseignés. La base de la pyramide étant constituée par le niveau Licence et le sommet par le niveau Doctorat. Le Master serait le niveau intermédiaire. Nous concluons et dégageons des perspectives dans la quatrième section.

\section{Méthodologie}

Nous considérons le cas de pratiques pédagogiques (enseignements et évaluations) à caractère scientifique. Dans la classe, l'enseignant échange des informations [à travers des séances de cours Magistraux (CM), de Travaux Dirigés (TD) et de Travaux Pratiques (TP)] avec des apprenants. Ces échanges d'informations sont véhiculés par des ondes sonores. La parole (question 
posée par exemple) de l'enseignant est nécessairement codée au sortir de sa bouche (émetteur) pour être réceptionnée au niveau de l'oreille de l'apprenant (récepteur) via son tympan. Enfin, l'information est décodée au niveau du cerveau (centre biologique de décodage, de traitement, de compréhension et de restitution des informations) de l'apprenant. Les processus d'échanges d'informations entre enseignants et apprenants peuvent être bien décrits dans le cadre de la mécanique quantique basée sur la notion fondamentale d'onde. Pourquoi un muet n'enseigne-t-il pas ? Parce que tout simplement il ne peut pas émettre des sons (ondes sonores) sous-tendus par un langage articulé. Ainsi, nous posons que dans une classe, les séances d'enseignementapparentage sont des processus d'émission (par l'enseignant) et d'absorption (par les apprenants) d'informations véhiculées par des ondes sonores. Il faudra y intégrer les interactions apprenants-apprenants (émission et absorption d'informations entre apprenants). Or, dans les systèmes atomiques, les processus d'absorption et d'émission d'ondes lumineuses (photons) ont lieu entre des niveaux d'énergie quantifiés. En assimilant le niveau d'énergie à un niveau d'enseignement-apprentissage et les électrons aux apprenants (Sakho, 2011), on comprend aisément qu'un apprenant passe (transite) d'une classe à une autre par «absorption » (maitrise, assimilation) de connaissances tout comme un électron passe (transite) d'un niveau d'énergie à un autre par absorption d'énergie. Cette présente étude est basée sur cette analogie. Comme nous l'avons souligné dans l'introduction, nous adoptons dans ce travail la démarche analogique à partir d'une situation-source (système atomique) et une situation-cible (système pédagogique). Il s'agit d'établir des relations d'équivalence entre les éléments des situations-source et cible selon le modèle indiqué dans le tableau 1.

Tableau 1 : Relations d'équivalence entre les éléments des situations-source et cible.

\begin{tabular}{|c|c|c|}
\hline & $\begin{array}{l}\text { situation-source } \\
\text { système atomique }\end{array}$ & $\begin{array}{c}\text { situation-cible } \\
\text { système pédagogique (école, } \\
\text { université) }\end{array}$ \\
\hline \multirow{5}{*}{ Éléments } & électron & apprenant \\
\hline & noyau & enseignant \\
\hline & niveau d'énergie & niveau d'enseignement-apprentissage \\
\hline & transition électronique & passage en classe supérieur \\
\hline & ionisation & changement de cycle \\
\hline
\end{tabular}

Ce tableau analogique permet de passer à une représentation géométrique qui frappe plus l'esprit (fig.1).Les petits cercles dans le cas de la situation source représentent des électrons. Les transitions sont indiquées par des flèches ascendantes. 


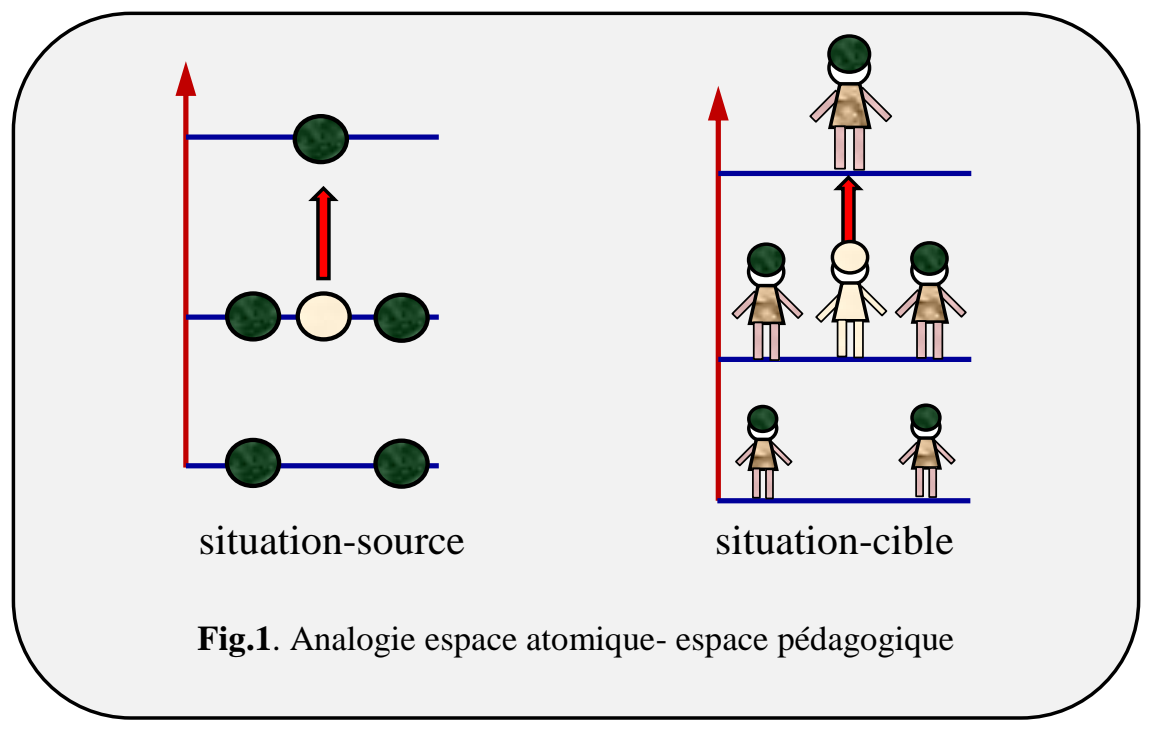

\section{Résultats et discussions}

\section{1. «Principe 7 » de la pédagogie}

\subsection{1. «Quantification » des niveaux d'enseignement-apprentissage :}

Nous nous proposons de montrer que les niveaux d'enseignementapprentissage dans les établissements scolaires et universitaires sont discrets et sont au nombre exact de 7 . Ce c'est que nous vérifions sur la figure 2 ciaprès dans le cas du système francophone. Nous considérons le système LMD actuellement en vigueur. Le système Fouchet est l'ancien système organisant les cursus dans l'enseignement supérieur. En effet, l'ancienne réforme française de 1967 à savoir la Réforme Fouchet, avait institué la licence en trois ans, la maîtrise en un an et le Diplôme d'Études Approfondies (DEA) en deux ans. Puis suivent le Doctorat de troisième cycle et le Doctorat d'État. Ce dernier diplôme était indispensable en Lettres et en Sciences pour accéder au grade de Professeur. De plus, cette architecture comportait différents autres diplômes comme les diplômes d'Études Supérieures (DES) et les Diplômes d'Études Supérieures Spécialisées (DESS). Depuis le processus de Bologne de 1999, le système LMD (Licence, Master et Doctorat) a vu le jour. Cette réforme a pour objet la création d'un espace européen de l'enseignement supérieur soutenue par l'Union Européenne (Karin, 2009 ; Cedies, 2004 ; CRID, 2016).Cette réforme s'inscrit dans la perspective d'une harmonisation des diplômes pour assurer la mobilité des étudiants et des enseignants et une reconnaissance mutuelle des diplômes entre pays européens. Dans le cadre du CAMES (Conseil Africain et Malgache de l'Enseignement Supérieur), les pays de l'UEMOA possèdent un espace universitaire commun. L'adoption du système LMD aussi bien dans l'espace européen que dans l'espace africain, a 
permis une simplification de l'architecture des repères et des diplômes en ramenant ceux-ci à trois: Licence, Master et Doctorat, avec comme conséquence majeure la suppression de la thèse de Doctorat d'État, un facteur bloquant dans la carrière des enseignants du Supérieur en Sciences et en Lettres, par le temps que cette thèse prend pour sa réalisation. En comparant le système Fouchet et le système LMD (fig.2), on constate qu'une formation universitaire débouchant au doctorat unique ne peut être complète que si l'étudiant fait une formation complémentaire postdoctorale. Ce qui concilie alors le système Fouchet (Fig.2.c) et le nouveau système LMD (fig.2.d). Nous avons adopté dans ce travail l'approximation des niveaux d'enseignementapprentissage discrets équidistants. Mais ces niveaux ne sont pas équidistants puisque les contenus des programmes ou des modules ne sont pas les mêmes d'un niveau à un autre. Pour faire image, la quantité d'énergie (effort) à fournir pour faire passer un apprenant du préscolaire au Cours Initiation (CI) est différente de l'effort à fournir pour faire passer par exemple un apprenant du Cours Moyen Élémentaire $1^{\mathrm{e}}$ année (CEM1) au Cours Moyen Élémentaire $2^{\mathrm{e}}$ année (CEM2). Il en est de même des niveaux d'enseignement-apprentissage au moyen-secondaire et au Supérieur.

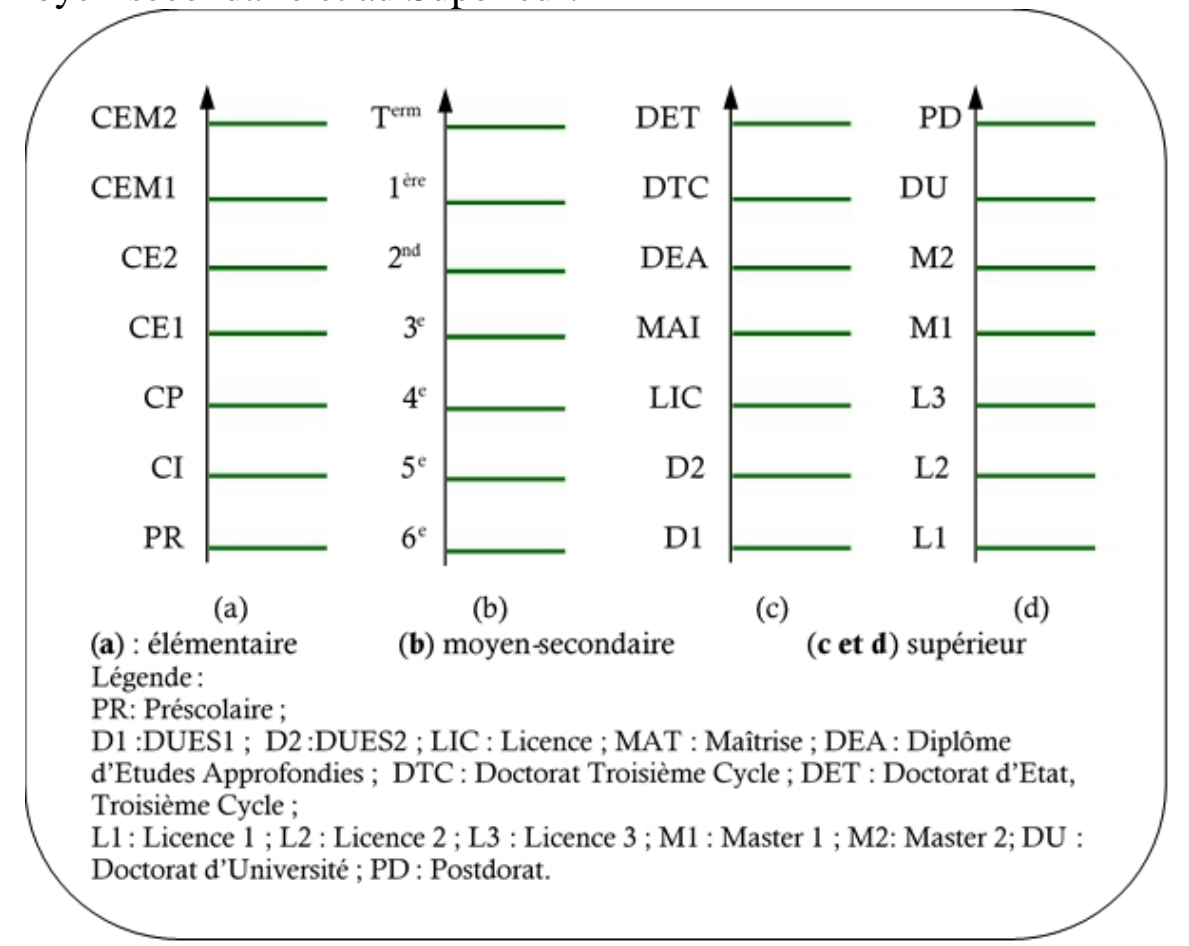

Fig.2. Quantification des niveaux d'enseignement scolaires et universitaire.

En outre, il est intéressant d'expliquer la notion de dégénérescence des niveaux d'enseignement scolaires et universitaires des apprenants sur la base 
de la figure 1. Considérons à cet effet la figure 3 en ne tenant compte que du niveau de la classe de Seconde. Il est clair que les trois élèves qui passent en Seconde pour la première fois, n'ont pas le même niveau d'instruction que leurs deux camarades redoublants. On dira alors que la classe de Seconde est « dégénérée », c'est-à-dire que, pour le même niveau d'instruction, correspondent plusieurs «états » de connaissances différents. Notons que le niveau préscolaire est trois fois "dégénéré ». Il lui correspond les sousniveaux Petite Section (PS), Moyenne Section (MS) et Grande Section (GS). Cependant, le niveau fondamental des systèmes atomiques hydrogénoïdes est seulement deux fois dégénéré. Les sous-niveaux sont déterminés par les valeurs possibles du nombre quantique magnétique de spin $m_{\mathrm{s}}=+1 / 2$ et $m_{\mathrm{s}}=$ - 1/2. On devrait donc considérer deux sous-niveaux pour le préscolaire : PS (à partir de 4 ans) et GS (à partir de 5 ans). Á l'âge de 6 ans, l'enfant accède en classe de CI. Entre 0 et 3 ans, l'enfant a plutôt besoin de la chaleur parentale: il doit rester «blotti » dans les doux bras de sa maman pour humer la douceur de la tendresse maternelle.

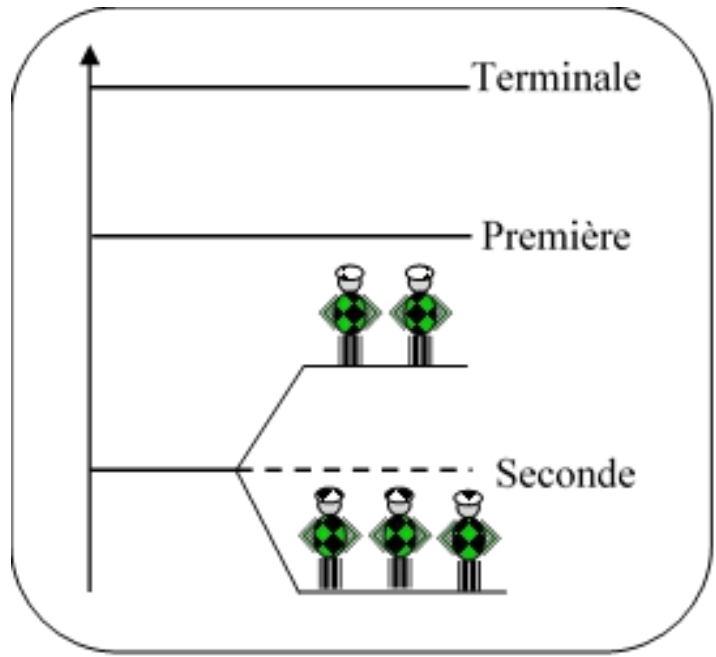

Fig.3. «Dégénérescence » du niveau fondamental du cycle secondaire (niveau Seconde). Les deux redoublants n'ont pas le même niveau d'instruction que les trois élèves nouvellement admis en Seconde (Sakho, 2011).

\subsection{2. Équivalents pédagogiques des états fondamental, excités et ionisé des systèmes atomiques, nombre de diplômes de l'élémentaire au Supérieur}

Dans un système atomique hydrogénoïde, l'état de plus basse énergie est l'état fondamental caractérisé par le nombre quantique principal $n=1$. Puis suivent les états électroniques excités pour $1<n<\infty$. L'état ionisé correspond à $n=\infty$. Notons que l'électron appartient au système atomique tant que $1 \leq n$ $<\infty$. Pour $n=\infty$, l'électron est expulsé du système qui se retrouve ainsi dans un état ionisé. Dans ce cas, l'électron n'appartient plus au système atomique. 
Dans la situation pédagogique, l'apprenant quitte le système scolaire ou universitaire après avoir obtenu le diplôme préparé pour le cycle considéré. Le nombre de diplômes préparés de l'élémentaire au Supérieur est égal à 7 (dans le système francophone) :

1. Certificat d'Études Primaires Élémentaires (CEPE);

2. Diplôme de Fin d'Études Moyen (DFEM) ou Brevet de Fin d'Études Moyen (BFEM) ;

3. Baccalauréat (BAC);

4. Diplôme d'Études Universitaires Scientifiques (DUES) ou Littéraires (DUEL)

5. Licence

6. Master

7. Doctorat

Dans le système LMD, le DUES ou le DUEL peut être délivré à l'étudiant ayant validé ses semestres en L1 et L2. Mais, très souvent, les étudiants préfèrent obtenir un diplôme de Licence au sortir de la L3 après avoir obtenu 180 crédits. Ainsi, les niveaux de préparation des diplômes sont discrets ou «quantifiés ». Á l'élémentaire, le niveau « ionisé » correspond au CEM2. L'élève ayant obtenu son CEPE quitte le cycle élémentaire. Il peut intégrer un nouveau cycle : le moyen. De même, l'élève ayant obtenu son BFEM quitte le cycle moyen (collège) pour intégrer un nouveau cycle : le secondaire. Après l'obtention du BAC, l'apprenant quitte le secondaire. Il peut être admis dans un autre cycle de formation : l'université. Enfin, après avoir obtenu son $7^{\mathrm{e}}$ diplôme : le Doctorat, l'apprenant quitte l'université pour s'insérer dans le monde du travail. Si avec son grade de Docteur, il est recruté dans une université, il y revient en tant qu'enseignant-chercheur et non en tant qu'apprenant.

\subsubsection{Journée normale et journée continue}

Avant l'instauration du régime de la journée continue (RJC) dans les établissements scolaires avec la création des cantines scolaires, l'apprenant partageait son temps de travail en temps de présence à l'école entrecoupé d'un temps de présence à la maison. Ceci correspondait au régime de la journée normale $(\mathrm{RJN})$ où l'apprenant passait au total $\mathbf{7}$ heures à l'école du matin au soir avec une pause effectuée à la maison (fig.4.a). Dans le cas du RJC, l'apprenant passe tout son temps à l'école sans retourner à la maison (fig.4.b). Mais, force est de constater que le RJC contribution à baisser la performance des apprenants. En effet, dans certains établissements scolaires au Sénégal, lors des courtes pauses de $15 \mathrm{~min}$, les apprenants passent plus de temps avec leurs téléphones portables et ne disposent pas assez de temps pour bien se restaurer et se prélasser. 


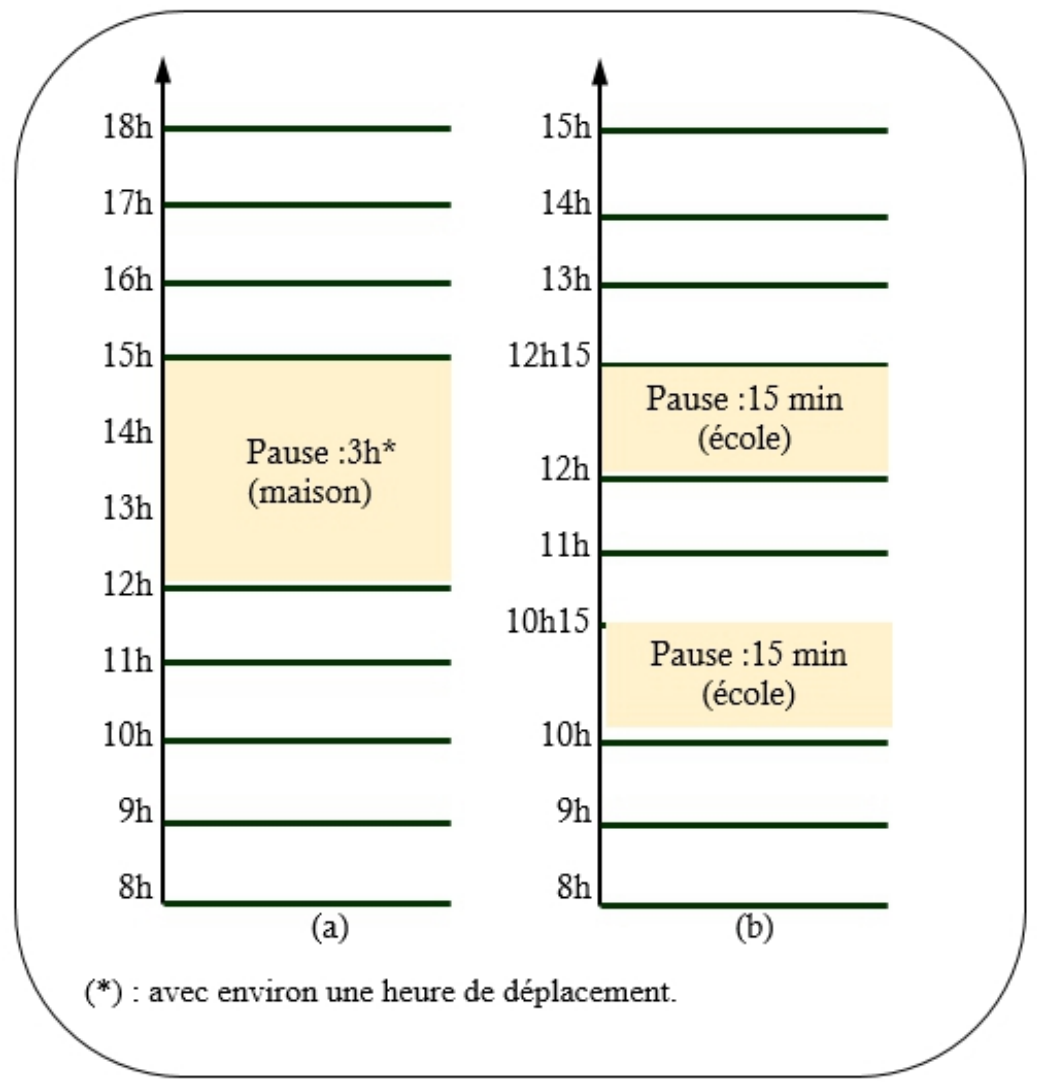

Fig.4. Régimes de journée normale (a) et de journée continue (b).

Nombre de chefs d'établissements conscients de ce phénomène ont inscrit dans leurs règlements intérieurs l'interdiction du téléphone portable à l'école. Lors des pauses à l'école, les apprenants sont laissés à eux-mêmes sans aucun contrôle. Certains ne mangent pas à leur faim (parfois ils se suffisent à prendre tout juste un bout de pain et une tasse de café en 15 min seulement). Ils ne peuvent pas se reposer, et s'ils veulent dormir, ils ne peuvent que se compter de tables - bancs par trop inconfortables (nombre d'élèves dorment durant les cours de l'après-midi sous l'effet de la fatigue et de la faim). Par contre, avec le RJN, l'apprenant descend à midi à la maison. Il a le temps de manger en famille, de prendre une douche, de faire une petite sieste pour se soulager de la fatigue induite par les séances d'enseignement-apprentissage de $8 \mathrm{~h}$ et $12 \mathrm{~h}$. De plus, ses parents pourront s'informer sur son état de santé et contrôler s'il a des exercices à faire pour l'après-midi. Voici les quelques avantages du RJN appliqué par nombre d'établissements du moyen-secondaire au Sénégal par exemple. Ces établissements disposent de bus ou de minicars de transport pour faciliter les déplacements des apprenants entre leurs domiciles et leurs établissements scolaires. On notera que le temps de pose est très court dans le RJC (30 min au total): les élèves doivent manger et se reposer 
en 30 min. Même si la durée de la pause dans le RJN intègre le temps de transport aller-retour domicile-établissement solaire, les apprenants ont au moins 30 à 45 min de sieste. Nous nous rappelons d'une situation de malaise quotidienne qui affectait l'un de nos élèves au lycée de Bambey au Sénégal où nous exercions en qualité de professeur de Sciences Physiques entre 2002 et 2008. Ayant remarqué que l'un de ses élèves somnolait souvent durant son cours de l'après-midi, le professeur l'interpella: tu n'arrives pas à suivre mon cours normalement parce que tu passes tout ton temps à dormir ! L'élève répondit : comment suivre un cours quand on a le ventre creux ? C'est à peine qu'on pouvait retenir ses larmes en entendant la réponse de l'élève. Cet élève n'est pas un cas isolé. Ils sont nombreux à vivre ces situations dramatiques. Comment un élève peut-il manger à sa faim et se reposer durant les courtes pauses perlées de 15 min entre 8 h et 15 ? Le RJC met les élèves dans des situations inconfortables induisant des pertes de performance. Ce qui justifierait en partie les déperditions scolaires observées: échecs massifs, abandons, etc. Contrairement au RJC, le RJN correspondant $7 \mathrm{~h}$ de travail de l'apprenant. Il lui permet de suivre normalement ses études tout en lui permettant de bien se restaurer et de se reposer. Pour éliminer la fatigue que pourrait induire le transport, il serait plus judicieux que l'apprenant s'inscrive dans un établissement de proximité. Les va-et-vient journaliers correspondant à 1 h 30 min de marche environ, l'établissement de l'apprenant pourrait être situé à $300 \mathrm{~m}$ de son domicile en supposant qu'il effectue $10 \mathrm{~m}$ de marche par min. Deux établissements scolaires de proximité seraient alors distants de 600 m. Le schéma des activités de l'apprenant en 7 h30 et 18 h est le suivant :

1/ quitte le domicile familiale à $7 \mathrm{~h} 25$ :

$2 /$ arrive à l'école à $7 \mathrm{~h} 50$;

3 / fait cours de $8 \mathrm{~h}$ à $12 \mathrm{~h}$ (y compris la récréation) ;

$4 /$ revient à la maison à $12 \mathrm{~h} 30$;

$5 /$ prend son repas et son dessert entre $12 \mathrm{~h} 30$ et $13 \mathrm{~h}$;

6/ prend une douche et fait une sieste entre 13 et $14 \mathrm{~h} 30$.

$7 /$ reviens à l'école à $14 \mathrm{~h} 50$.

Á la descente à 18h, l'apprenant n'est plus lié à l'école : il peut décider de faire un crochet chez un parent pour lui dire bonjour ou même partager avec lui le dîner avant de rentrer à la maison. On notera au moins quatre avantages du RJN : marche de l'apprenant pendant 1 h 30 min environ par jour; restauration en toute quiétude; temps de repos suffisant pour reprendre les cours de l'après-midi en forme ; contrôle de l'état de santé de l'apprenant et de ses activités pédagogiques par les parents. On perd tous ces avantages en instaurant le RJC. Notons que l'apprenant gagnerait plus de temps s'il se déplaçait en vélo. La marche et le vélo, très bénéfiques pour le corps et l'esprit (Choquette, 2017 ; Nénard, 2017) sont préférables aux bus et 
aux minicars de transports élèves qui peuvent polluer l'air par le rejet de gaz toxiques (monoxyde d'azote $\mathrm{NO}$, dioxyde d'azote $\mathrm{NO}_{2}$, monoxyde de carbone $\mathrm{CO}$, dioxyde de carbone $\mathrm{CO}_{2}$, dioxyde de soufre $\mathrm{SO}_{2}$, etc.) émanant de la combustion de l'essence ou du gasoil. Dans le cadre pédagogique, les travaux du Professeur Stephen Cunnane du Département de médecine de l'Université de Sherbrooke montrent que (...) la marche améliorerait quelque peu les capacités cognitives, en particulier la vitesse de traitement de l'information. Le cerveau s'active (Choquette, 2017). Quant au vélo, sa pratique permet une meilleure irrigation et approvisionnement en sang donc une meilleure oxygénation des neurones, stimulant ainsi leur croissance et diminuant leur dégénérescence ; le cerveau est alors boosté (Nénard, 2017). Ainsi, un cas idéal serait de créer des établissements scolaires de proximité, loin des zones à risque (ravins, décharges d'ordures, usines, réseaux trafics denses, etc.) et accessibles à pied ou par vélo via des pistes cyclables et/ou des routes en sens unique.

\subsubsection{Principe d'exclusion de Pauli \& principe d'indiscernabilité : occupation des tables-bancs \& tenue scolaire}

Nous nous proposons de préciser le nombre correct d'apprenants par table-banc dans une classe pédagogique par analogie avec l'occupation des états quantiques par les électrons atomiques. En vertu du principe d'exclusion de Pauli, un état électronique ne peut contenir au maximum que deux électrons. Par analogie, le nombre d'apprenants par table-banc dans une classe pédagogique doit être égal au maximum à deux. Le fait de mettre trois apprenants sur le même table-banc est inacceptable. On se rend rapidement compte de cette aberration lorsqu'on se propose d'évaluer de tels apprenants. Á titre d'exemple illustratif, comparons la configuration électronique $1 s^{2} 2 s^{2} 2 p^{6}$ de l'atome de néon contenant dix électrons et la répartition de dix apprenants sur les premières rangées d'une classe donnée (fig.5).

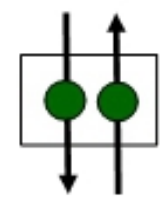

$1 \mathrm{~s}^{2}$
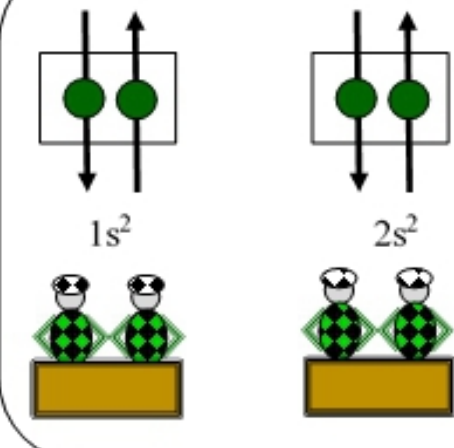

$2 s^{2}$
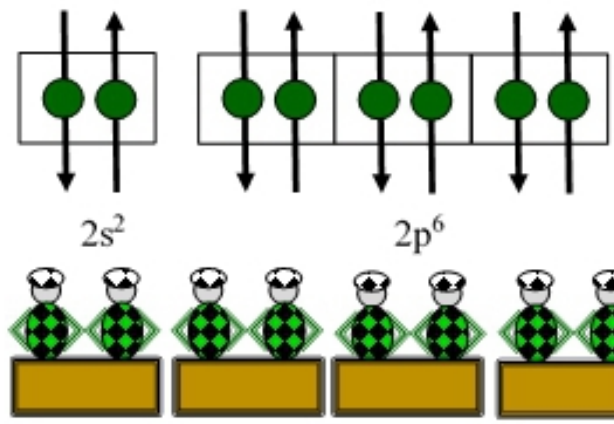

$2 \mathrm{p}^{6}$
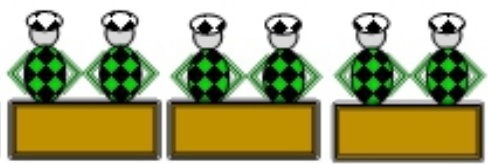

Fig.5. Analogie entre le modèle en case de la configuration électronique des atomes et l'occupation correcte des tables-bancs par des élèves (Sakho, 2011). 
Les électrons occupent les cases quantiques sans aucune « contraintes ». On doit donc laisser les apprenants s'assoir deux à deux par affinité. L'école étant à la fois scolarisant (volet pédagogique) et socialisant (volet affectif), on ne doit pas imposer deux apprenants à partager le même table-banc car ils peuvent s'exclure mutuellement pour des raisons qui leurs sont propres. Cet effet de gêne mutuelle impactera leurs concentrations durant le cours. De plus, les électrons étant indiscernables (symbolisés par des cercles de même couleur), les apprenants devraient aussi être «indiscernables » : la tenue scolaire permet de satisfaire le principe d'indiscernabilité dans le système pédagogique. Sur la figure 5, les dix apprenants sont « indiscernables »: on ne sait pas qui est issu d'une famille nantie ou d'une famille démunie.

\subsubsection{Interaction électromagnétique et pédagogie active}

Dans le tableau 1 précédent, nous avons indiqué les relations d'équivalence entre les éléments des situations source (système atomique) et cible (système pédagogique). Nous cherchons à analyser la nature des interactions entre les acteurs (enseignant et apprenants) lors d'une séance enseignement - apprentissage. De toute évidence, la transmission des connaissances et l'évaluation des objectifs pédagogiques sont du ressort de l'enseignant. Quel comportement doit alors adopter l'apprenant lors d'une séance enseignement - apprentissage ?

On distingue deux grandes familles de méthodes pédagogiques : la pédagogie expositive ou passive et la pédagogie active qui englobe en son sein les formes de pédagogie démonstrative, interrogative, de découverte, expérimentale et la pédagogie par projet (Sakho, 2018). La pédagogie expositive, passive, transmissive ou encore magistrale est la première des méthodes pédagogiques. L'enseignant transmet ses connaissances sous forme d'un exposé : c'est le cours magistral qui laisse peu de place à l'interactivité avec l'apprenant qui ne fait que subir sans pouvoir réagir. Le cours magistral tel que défini, ne permet pas à l'apprenant de s'approprier les connaissances transmises, sauf s'il est articulé à d'autres activités pédagogiques telles que les Travaux Dirigés (TD), les Travaux Pratiques (TP), les sorties pédagogiques (SP) etc., qui permettront un véritable travail cognitif. La pédagogie expositive est révolue : elle doit être jetée dans les oubliettes. La pédagogie active se réfère historiquement et principalement au pédagogue suisse Adolphe Ferrière (1879-1960) qui a été parmi les premiers à utiliser l'appellation école active dans sa publication intitulée «la Pratique de l'école active » parue en 1924 (UNESCO, 1993 ; Wikipédia, 2018). Dans cette forme de pédagogie, le rôle de l'enseignant se déplace progressivement du contenu à enseigner vers l'apprenant qui reste au cœur des interactions et devient le 
principal acteur de son apprentissage. Aux interactions enseignant-apprenants s'ajoutent alors les interactions apprenants-apprenants.

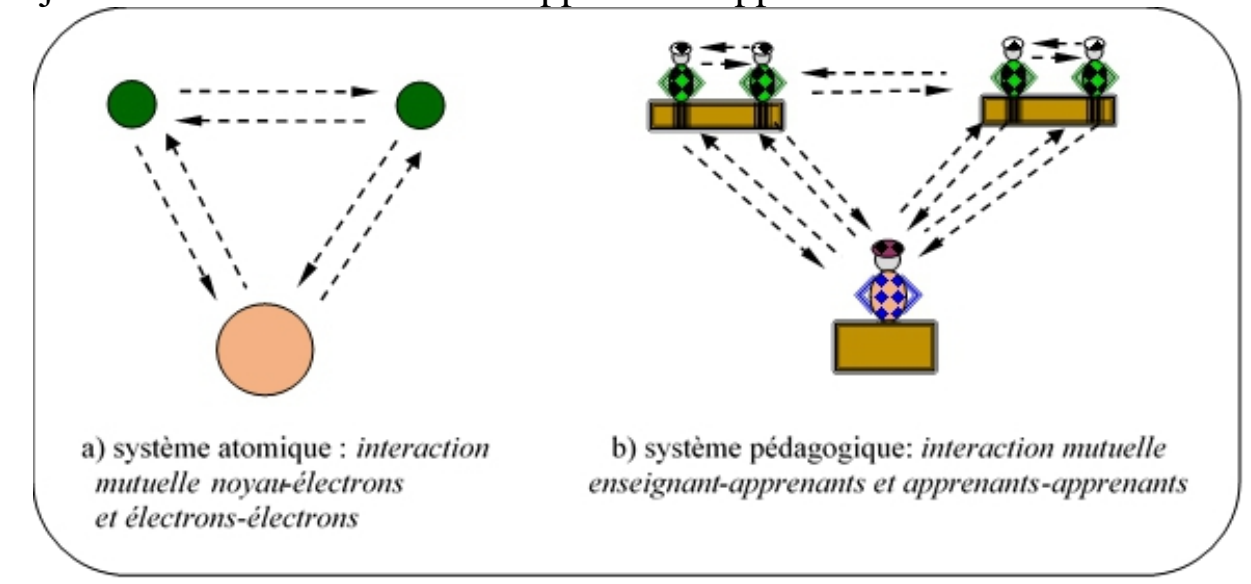

Fig.6. Mise en évidence de la pédagogie active: analogie des interactions noyau-électrons et électrons-électrons dans les systèmes atomique et enseignant-apprenants, apprenantsapprenants dans les systèmes pédagogiques.

Pourtant, dès la découverte du noyau par le physicien anglais Ernest Rutherford (1871-1937) en 1911, le modèle planétaire de l'atome été sorti de terre. D'après ce modèle, l'atome est constitué d'un noyau plongé dans un nuage d'électrons. Mais dans un atome, les interactions coulombiennes sont du type noyau-électrons et électrons-électrons. Dans une situation pédagogique ce schéma d'interactions coulombiennes correspond aux interactions enseignant-apprenants et apprenants- apprenants. Cette analogie est beaucoup plus frappante si on l'analyse dans le cadre de la théorie des quatre interactions fondamentales: interaction gravitationnelle traduisant l'attraction mutuelle entre les corps matériels non chargées, l'interaction électromagnétique qui est à l'origine de la cohésion entre le noyau et les électrons, et qui plus généralement régit les interactions entre particules chargées, l'interaction nucléaire forte assurant la cohésion entre les nucléons (protons et neutrons) dans le noyau atomique et l'interaction nucléaire faible responsable de certaines désintégrations nucléaires comme la désintégration $\beta$ (Grossetête, 1985). Ces interactions résultent d'échanges de particules appelée conventionnellement «bosons » vecteurs : gluon pour l'interaction forte, photon pour l'interaction électromagnétique, bosons $W$ et $Z$ pour l'interaction faible et l'hypothétique « graviton » pour l'interaction gravitationnelle. Dans le cadre de cette étude seule l'interaction électromagnétique avec échange de photon retiendra notre attention. Ce qui permet alors de faire le schéma analogique illustré sur la figure 6 . Cette analogie montre que l'on pouvait mettre en œuvre la pédagogie active dans les systèmes scolaires et universitaires dès l'élaboration du modèle planétaire de l'atome en 1911, soit donc 13 ans avant la théorisation de la «Pratique de l'école active » 
d'Adolphe Ferrière. Remarquons que dans les systèmes atomiques, le noyau occupe le centre de l'atome et les électrons sont distribués dans un nuage entourant le noyau. Dans le cadre pédagogique, il serait intéressant d'adopter une disposition où l'enseignant est entouré par les apprenants. Cette approche très innovante serait possible dans le cadre d'une pédagogie assistée par ordinateur : enseignant et apprenants disposent chacun d'un microphone et d'un ordinateur avec un accès à un réseau WIFI permanant, tous les ordinateurs étant bien évidement interconnectés (voir figure 10, paragraphe 3.1.9 pour plus de détails à ce sujet).

\subsection{6. «Principe 7 » de la pédagogie}

D'après ce qui précède, nous avons montré par analogie avec les systèmes atomiques, que les niveaux d'enseignement-apprentissage dans les établissements scolaires et universitaires sont discrets et sont au nombre exact de 7. De plus, le RJN correspondant exactement à $7 \mathrm{~h}$ de travail de l'apprenant. De même, dans l'espace francophone, le nombre de diplômes préparés de l'élémentaire au Supérieur est égal à 7. Or, comme nous l'avons précisé en introduction, toutes les activités de l'homme ont lieu en 7 jours répétés. Le chiffre 7 se révèle donc être le « chiffre de la pédagogie ». Ce constat nous amène à énoncer ce que nous appelons le « principe 7 » de la pédagogie :

«Dans un système pédagogique les nombres d'éléments constitutifs sont au nombre de 7 ou un multiple de 7. »

Les éléments constitutifs d'un système pédagogiques sont les suivants :

- nombre de niveaux d'instruction (ou niveaux d'enseignementapprentissage) ;

- nombre d'heures de travail apprenants ;

- nombre d'heures de travail enseignant;

- nombre d'heures de travail personnel administratif ;

- nombre de disciplines/classe ;

- nombre de classes physiques (un multiple de 7).

Notons une exception pour le nombre de diplômes. Ici on doit considérer tout le parcours de l'apprenant de l'élémentaire à l'Université, soit au total 7 diplômes (voir paragraphe 3.1.2).

De plus, le cas idéal dans le système scolaire serait qu'apprenants, enseignants et personnel administratif effectuent tous $7 \mathrm{~h}$ de travail par journée ouvrable. Toutefois, ce principe est difficilement applicable au Supérieur puisque le volume statutaire est corrélé au grade de l'enseignantchercheur. Au Sénégal par exemple, les volumes statutaires des PER (Personnel Enseignant et de Recherche) sont les suivants : 
- Maître Assistant (MA): 102 H /12 semaines soit ; 8 h30 min/semaine

- Maître de Conférences (MC) et Professeur Titulaire (PT): 60/12semaines, soit $5 \mathrm{~h} /$ semaine ;

Un cas idéal serait le suivant:

MA: activités pédagogiques: 7h/semaine réparties en $\mathrm{CM}$, TD et TP éventuellement

MC et PT: activités pédagogiques et d'encadrement : $7 \mathrm{~h} /$ semaine dont $5 \mathrm{~h}$ de $\mathrm{CM}$ et $2 \mathrm{~h}$ de séminaire pour les niveaux Master et Doctorant.

Toutefois, certains PER exercent en plus des activités pédagogiques et de recherches, des activités administratives : Chef de Département, Directeur d'UFR et Vice-Recteur. Les $7 \mathrm{~h}$ de travail préconisées dans l'espace universitaire ne concernent que les activités pédagogiques.

Par ailleurs, il serait intéressant de vérifier si le principe 7 de la pédagogie est satisfait pour ce qui concerne le nombre de disciplines. Considérons à cet effet la classe de Terminale $\mathrm{S}$ puis comparons le nombre de disciplines dans le cas de la France et du Sénégal.

En France, on distingue deux types d'enseignement en Terminale S :

- Enseignements spécifiques : Mathématiques, Physique-chimie, Sciences de la vie et de la Terre, Sciences de l'ingénieur, Histoire-Géographie, Philosophie ;

- Enseignements de spécialité : Mathématiques, Physique-chimie, Sciences de la vie et de la Terre, Informatique et sciences du numérique.

Source: Les programmes pour le cycle terminal (classes de première et de terminale). Texte de référence : arrêté du 12-6-2015, Bulletin officiel spécial $n^{\circ} 6$ du 25 juin 2015

https://www.eduscol.education.fr/cid46522/programmes-du-cycle-terminal-de-la-voiegenerale. html.

La comparaison du nombre de disciplines est indiquée dans le tableau 2.

Tableau 2. Comparaison du nombre de disciplines en Terminale $\mathrm{S}$ dans le système éducatif de deux pays francophones.

\begin{tabular}{|l|l|l|}
\hline Nbre & \multicolumn{1}{|c|}{ France } & \multicolumn{1}{|c|}{ Sénégal } \\
\hline & Enseignements spécifiques \& de spécialité & \multicolumn{1}{|c|}{ Enseignement général } \\
\hline 1 & Mathématiques & Mathématiques \\
\hline 2 & Physique-chimie & Sciences Physiques \\
\hline 3 & Sciences de la vie et de la Terre & Sciences de la Vie et de la Terre \\
\hline 4 & Sciences de l'ingénieur & Histoire- Géographie \\
\hline 5 & Informatique et sciences du numérique & Anglais \\
\hline 6 & Histoire- Géographie & Français \\
\hline 7 & Philosophie & Philosophie \\
\hline
\end{tabular}

NB : l'éducation Physiques et Sportives en classe de Terminale n'est pas une discipline que l'on enseigne en classe. Elle est donc exclue. 
Une comparaison des disciplines listées dans le tableau ci-dessus montre qu'il se pose un sérieux problème de niveau de compétences : un bachelier sénégalais issu de la série $\mathrm{S}$ et qui désire aller en France pour poursuivre ses études, est moins outillé que son camarade français. Les bacheliers sénégalais de la série $\mathrm{S}$ n'ont aucune notion ni sur les sciences de l'ingénieur ni sur les sciences numériques. Le baccalauréat ne doit pas être corrélé à une nation : il doit être un diplôme universel. D'où la nécessité d'avoir le même nombre de disciplines par classe ainsi que les mêmes intitulés pour les différentes matières enseignées. Le «principe 7 » de la pédagogie suggère 7 disciplines en Terminale (et dans toutes les classes) en France, au Sénégal et tout dans tout l'espace francophone. Les pays anglophones n'ont pas le même système éducatif que les pays francophones. Par exemple, les « A-Levels » (« niveaux avancés »), sont l'équivalent du Bac français pour les étudiants anglais (Despres, 2016). Un programme en Terminale $S$ qui serait commun aux candidats français et sénégalais serait le suivant :

1. Mathématiques;

2. Sciences Physiques ;

3. Sciences de la Vie et de la Terre ;

4. Sciences de l'ingénieur;

5. Informatiques et sciences numériques ;

6. Histoire-Géographie ;

7. Philosophie.

Dans le cas du Sénégal, les langues (Anglais \& Français) supprimées en Terminale $\mathrm{S}$ seront renforcées dans les programmes des classes antérieures (du Préscolaire à la classe de Première). Les apprenants disposeront alors suffisamment de temps pour maitriser et parler couramment l'anglais et le français avant d'arriver en Terminale S. Par ailleurs, il serait aussi intéressant d'étudier la possibilité de résoudre le problème que pose la surcharge des classes par application du « principe 7 » de la pédagogie. En effet, la surcharge des classes est considérée comme un véritable enfer par divers acteurs du secteur de l'éducation à travers le monde. Au Sénégal, les enquêtes réalisées par Baldé, Bop \& Faye, (2017) sont révélatrices. C'est auteurs ont montré qu' en plus du déficit de matériels pédagogiques ou manuels didactiques influant négativement sur les évaluations nationales, les salles de classe sont bondées de potaches. Les élèves s'entassent, comme des sardines, loin de la norme internationale de 45 élèves par classe. La pléthore d'apprenants dans les classes affecte le niveau des élèves. Á l'école élémentaire, les enseignants se sont lassés de tirer la sonnette d'alarme. Sous le couvert de l'anonymat, un enseignant a exprimé son amertume par rapport à leurs conditions de travail dans les salles qui ont des effectifs de plus 85 élèves. Selon lui, «ce problème 
est lié au fait que la banlieue dakaroise manque d'établissements publics». Dans certains collèges de la banlieue dakaroise, on note des classes croulant sous le poids des effectifs : 52 à 72 élèves par classe en $3^{\mathrm{e}}$. L'une des classes de $6^{\mathrm{e}}$ affiche 95 élèves sur la liste d'appel. Une élève décrit avec désolation ce qu'elle avait vécu en classe : « quand j'étais en classe de $6^{\mathrm{e}}$ nous étions au nombre de 92 élèves dans la salle. C'était vraiment atroce», nous confie une élève, tout en nous indiquant que «les professeurs renvoyaient automatiquement les bavards et les perturbateurs pour dégraisser la salle». Le cri du cour de Marianne, lycéenne, est poignant : « le fait de se retrouver dans une classe avec plus de 60 élèves est un frein pour nous. Il est difficile de se concentrer. Ils nous arrivent parfois de ne pas bien comprendre le cours» [...] «le professeur est obligé de gérer plusieurs choses à la fois. Il lui est difficile de repérer les élèves qui perturbent le cours». Kany, élève au même lycée et vêtue de son uniforme, abonde dans le même sens : «en tant qu'élève de Terminale, nous avons besoins de bien assimiler nos cours. Ce qui est incompatible avec ce surpeuplement dans les classes. Nos professeurs rencontrent d'énormes difficultés pour s'acquitter de leur tâche». Quant à Malick, il souligne que «le déficit de professeurs y est pour quelque chose. Car, parfois, les professeurs sont obligés de regrouper deux classes pour faire un cours. Ce qui dépasse totalement la norme. De même, en Côte d'Ivoire, les effectifs pléthoriques des classes ont été décriés par les acteurs du système éducatif. On notait en 2012 des effectifs de 100 à 130 élèves en classe de Terminale (Napargalé, 2012). En Tunis, nombre d'acteurs du secteur de l'éducation ont montré que la surcharge des classes impact sur la qualité des enseignements. Selon Héla, enseignante, « une classe surchargée aura un impact négatif sur l'apprentissage, notamment la démotivation de l'élève et de l'enseignant, la fraude, l'indiscipline, la crise de nerf de l'enseignant, les châtiments corporels, la violence, ..... La réduction de la taille d'une classe est une condition pour mettre en œuvre une pédagogie plus ou moins différenciée, elle est de surcroit, suffisante pour avoir un enseignement de qualité répondants aux attentes du corps enseignant ». Samia, jeune enseignant abonde dans le même sens : «au-delà de 30 élèves, cela devient compliqué, sauf dans une classe avec un bon niveau. Cela demande beaucoup plus d'énergie et d'autorité car il est de moins en moins évident de passionner les élèves ». Pour Amel, «l'idéal au primaire serait des classes de 24 élèves maximum. En primaire, une classe surchargé de 35, voire 42 élèves est un enfer ! On est obligé de leur demander une très grande attention. C'est très compliqué ». [...] « Dans un groupe plus restreint, il est possible d'avoir plus de flexibilité. Dans une classe surchargée, mieux vaut être bon élève ! Un élève en difficulté a plus de risque de couler, car l'enseignant, débordé, ne pourra plus lui consacrer assez de temps » (Bouaouina, 2017). Dans les 36 pays membres de l'OCDE (Organisation de coopération et de développement 
économiques), les effectifs varient d'un pays à un autre. La moyenne est de 22 élèves par classe au primaire. Pour l'Estonie, la république slovaque et la Slovénie, les effectifs moyens par classe sont inférieurs à 19. Des pays comme le Mexique et la République tchèque ont des effectifs moyens par classe inférieurs à 20 alors pour le Luxembourg, l'effectif moyen est de 16 élèves par classe (Bouaouina, 2017).

D'après ce qui précède, il y a d'importantes variantes sur le nombre d'élèves minimal que doit comporter une classe. Or, comme l'a si bien souligné Héla cité plus haut, la "réduction de la taille d'une classe est une condition pour mettre en æuvre une pédagogie plus ou moins différenciée, elle est de surcroit, suffisante pour avoir un enseignement de qualité.. ». Dans la perspective d'une éducation universelle mutualisée, on doit avoir les mêmes configurations des classes quelle que soit la nation considérée : mêmes nombre de classes pédagogiques par établissement, même nombre d'apprenants par classe, même programme d'enseignement, même nombre de disciplines par classe. Ainsi un apprenant sénégalais par exemple en classe de $\mathrm{CM} 2$, pourrait aller poursuivre ses études en classe de $6^{\mathrm{e}}$ dans n'importe quel pays du monde. Pour cela, tout apprenant de l'élémentaire doit être au moins trilingue (anglais, arabe et français). Analysons à présent les effectifs moyens par classe de l'élémentaire à l'Université sur la base du «principe 7 » de pédagogie. Pour cela, notons tout d'abord certaines normes internationales relatives à la superficie unitaire minimale par élève et par classe. Les surfaces des salles en fonction du nombre d'utilisateurs sont indiquées dans le tableau 3 (MENFP, 2010).

Tableau 3. Surfaces des salles en fonction du nombre d'utilisateurs.

\begin{tabular}{|l|c|c|c|}
\hline \multicolumn{1}{|c|}{ niveau } & surfaces des salles de classe & & nombre utilisateurs \\
\hline Préscolaire & $42 \mathrm{~m}^{2}$ & 35 élèves max. et 1 enseignant \\
\hline $1^{\mathrm{e}}$ année $-6^{\mathrm{e}}$ année & $50 \mathrm{~m}^{2}$ & 40 élèves max. et 1 enseignant \\
\hline $7^{\mathrm{e}}$ année $-12^{\mathrm{e}}$ année & $50 \mathrm{~m}^{2}$ & 40 élèves max. et 1 enseignant \\
\hline
\end{tabular}

En vertu du «principe 7 » de la pédagogie, le nombre d'élèves par classe doit être égal à un multiple de 7. Soit :

$\mathbf{7}(1 \times 7) ; \mathbf{1 4}(2 \times 7) ; \mathbf{2 1}(3 \times 7) ; \mathbf{2 8}(4 \times 7) ; \mathbf{3 5}(5 \times 7) ; \mathbf{4 2}(6 \times 7)$ et $\mathbf{4 9}(7 \times 7)$.

Une lecture des plages d'effectifs résultant de l'application du «principe 7 » de la pédagogie montre que le nombre 14 est un bon indicateur comparé à l'effectif moyen de 16 élèves par classe au Luxembourg. De même, le résultat 21 élèves par classe est en parfait accord avec la moyenne de 22 élèves par classe au primaire arrêtée dans les pays de l'OCDE. Les effectifs 35 et 42 sont de même en bon accord avec les résultats regroupés dans la dernière colonne du tableau 3. La surface minimale d'une salle standard étant de $50 \mathrm{~m}^{2}$ en raison de 1,20 $\mathrm{m}^{2}$ /élève (MENFP, 2010), les surfaces des salles en fonction du nombre d'utilisateurs résultant de l'application du «principe 7 » de la 
pédagogie sont indiquées dans le tableau 4. Les surfaces sont calculées sur la base de nombre d'élèves $\times$ espace élève par métré carré $\left(1,40 \mathrm{~m}^{2} /\right.$ apprenant $=$ $\left.7 \mathrm{~m}^{2} \times 0,2\right)$. Le facteur 1,4 est choisi pour avoir un résultat le plus proche d'une valeur entière multiple de dix. Par exemple, $14 \times 1,2=16,8 \mathrm{~m}^{2}$ alors que $14 \times 1,4=19,6 \mathrm{~m}^{2}$. De même $21 \times 1,2=25,2 \mathrm{~m}^{2}$ alors que $21 \times 1,4=29$, $6 \mathrm{~m}^{2}$.

Tableau 4. Surfaces des salles en fonction du nombre d'utilisateurs résultant de l'application du principe 7 de la pédagogie.

\begin{tabular}{|c|c|c|c|}
\hline \multirow[t]{2}{*}{ Niveau } & \multicolumn{2}{|c|}{ surface des salles } & nombre utilisateurs \\
\hline & exacte & corrigée & \\
\hline Préscolaire & $19,6 \mathrm{~m}^{2}$ & $20 \mathrm{~m}^{2}$ & 14 élèves max/1 enseignant \\
\hline Elémentaire (CI au CM2) & $29,4 \mathrm{~m}^{2}$ & $30 \mathrm{~m}^{2}$ & 21 élèves max/1 enseignant \\
\hline Moyen $\left(6^{\mathrm{e}}, 5^{\mathrm{e}}, 4^{\mathrm{e}}, 3^{\mathrm{e}}\right)$ & $39,2 \mathrm{~m}^{2}$ & $40 \mathrm{~m}^{2}$ & 28 élèves max/1 enseignant \\
\hline Secondaire $\left(2^{\text {nd }}, 1^{\text {ère }}, T^{\text {erm }}\right)$ & $49,0 \mathrm{~m}^{2}$ & $50 \mathrm{~m}^{2}$ & 35 élèves max/1 enseignant \\
\hline Université (Licence, Master) & $58,8 \mathrm{~m}^{2}$ & $60 \mathrm{~m}^{2}$ & 42 étudiants max/1 enseignant \\
\hline Université (Licence)* & $68,6 \mathrm{~m}^{2}$ & $70 \mathrm{~m}^{2}$ & 49 étudiants max/1 enseignant \\
\hline
\end{tabular}

*, amphithéâtre de 49 étudiants en Licence 1 et 2 où les effectifs sont les plus élevés. Il est envisageable des amphithéâtres de $\mathbf{2 1 0}(30 \times 7)$ étudiants. Toutefois, plus l'effectif est élève plus la tâche est difficile pour l'enseignant. C'est un véritable enfer de corriger 210 copies. Un effectif de 49 étudiants est très raisonnable. Il est généralement admis qu'un nombre faible d'élèves par enseignant se traduit par des classes moins peuplées permettant à l'enseignant de s'occuper davantage de chaque élève, ce qui peut contribuer à long terme à de meilleurs résultats (UNESCO, 2018). Les amphithéâtres de plus de 49 places pourraient réservés à des manifestations scientifiques telles que séminaires, conférences, colloques, etc. Il est important de tenir compte du ratio élèves /enseignant pour un enseignement de qualité en classe. Ce ratio est défini comme le nombre moyen d'élèves par enseignant dans un niveau d'enseignement donné et pour une année scolaire donnée, basé sur le nombre d'élèves ainsi que celui des enseignants. Il est calculé en divisant le nombre total des élèves inscrits dans un niveau spécifié d'enseignement par le nombre des enseignants au même niveau (UNESCO, 2018).

\subsubsection{Architecture pyramidale du système LMD}

De façon générale, les niveaux d'énergie des systèmes atomiques se resserrent avec l'accroissement du nombre quantique principal $n$. Autrement dit, les écarts énergétiques entre les niveaux d'énergie diminuent quand $n$ augmente. Du point de vue pédagogique, la diminution des écarts énergétiques correspondrait à la diminution du nombre de modules quand le niveau d'enseignement-apprentissage de l'apprenant augmente. Par exemple, à l'Université, lorsque le niveau d'instruction augmente, l'étudiant tend vers sa spécialisation (Doctorat). L'exemple de la filière MPCI est concluant. Un étudiant inscrit en L1 MPCI, pourra opter pour une Licence 2 PC puis pour une Licence 3 Physique ou Chimie. On voit à travers cet exemple, que la base de la pyramide se rétrécit lorsque le niveau de la Licence augmente, ce qui correspond bien à une diminution du nombre de modules à enseigner. De même, le nombre de modules en Master Physique est différent du nombre de 
modules en Licence Physique. Sinon une correction est nécessaire sachant que l'étudiant tend vers une spécialisation. Au Doctorat, le sujet de thèse porte bien évidement sur une thématique de l'un des modules traités généralement en Master 2. Ce qui justifie l'architecture pyramidale du système LMD (fig.7). De plus, la Licence, le Master et le Doctorat sont validés après que l'étudiant ait capitalisé respectivement 180, 120 et 180 crédits. Mais force et de noter qu'il manque une cohérence dans cette progression qui ne correspond pas à l'architecture pyramidale préconisée dans ce travail. Il serait plus judicieux de trouver une relation mathématique entre les crédits alloués pour satisfaire le schéma illustré sur la figure 7. Constatons d'abord que :

$\mathrm{L} / \mathrm{M}=3$ niveaux $/ 2$ niveaux $=3 / 2=180$ crédits $/ 120$ crédits.

En considérant les niveaux Master et Doctorat, on obtient :

$\mathrm{M} / \mathrm{D}=2$ niveaux $/ 1$ niveau $=2 / 1 \neq 120$ crédits $/ 180$ crédits $=2 / 3=1 /(3 / 2)$.

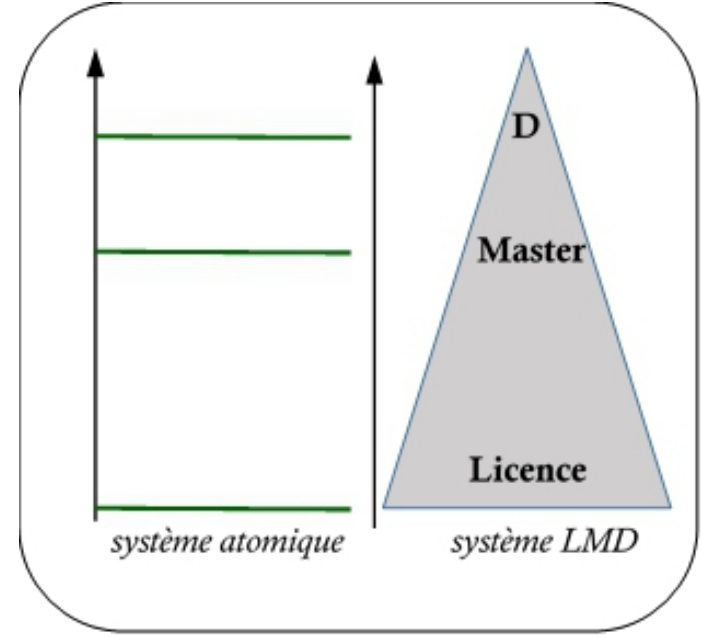

Fig.7. Architecture pyramidale du LMD

Il semblerait donc que le rapport 3/2 doit être utilisé pour fixer le nombre de crédits pour chaque niveau. Au sortir du processus de Bologne de1999, les matières à enseigner sont organisées en Unité d'Enseignement (UE). Á chaque UE correspond un temps de travail bien déterminé appelé crédit ou E.C.T.S (European Credit Transfert System). Un crédit vaut $20 \mathrm{~h}$ de travail réparti en temps de présence aux enseignements et en temps de travail personnel de l'étudiant (TPE). Un semestre correspondant à 30 crédits, les six semestres du Doctorat (trois ans) correspondent alors à $30 \times 6=180$ crédits. Mais le temps de travail en présentiel de l'étudiant en L ou en M est différent du temps de temps de travail de l'étudiants en thèse. Une thèse résulte d'un travail personnel. Le nombre de crédit alloués au niveau $\mathrm{D}$ donc être revu à la basse sur la base du rapport constant : $\mathrm{L} / \mathrm{M}=\mathrm{M} / \mathrm{D}=3 / 2$. En conservant les 180 crédits en L, on obtient le système 180/120/80 crédits pour L/M/D. On 
voit bien que cette progression est du type pyramidal (diminution du nombre de crédits de la Licence au Doctorat). Mais les 80 crédits en D semblent être inacceptables. On note une diminution de $100=180-80$ crédits soit au totale $2400 \mathrm{~h}$ de travail du doctorat sur les 3 années de formation doctorale. Cependant, la progression 180/120/80 correspondant au rapport $\mathrm{L} / \mathrm{M}=\mathrm{M} / \mathrm{D}=$ $3 / 2$ est parfaitement acceptable à condition de redéfinir le volume horaire affecté à un crédit pour le niveau D. Mais remarquons tout d'abord qu':

1. il existe un enseignement-apprentissage en Licence et en Master; par contre il n'existe aucun enseignement-apprentissage (évalué à travers des devoirs ou des examens) en Doctorat ;

2. les enseignements-apprentissages en Licence et en Master sont sous-tendus par des séances de CM, de TD et éventuellement de TP; il n'existe ni CM, ni TD, ni TP en Doctorat ;

3. il existe au moins des niveaux d'enseignement-apprentissage en Licence et en Master ; le Doctorat est unique.

D'après ces trois éléments de comparaison, il est clair qu'on ne peut comparer le Doctorat au Master encore moins à la Licence. Pourtant, l'étudiant effectue le même volume de travail $(180 \times 20 \mathrm{~h}=3600 \mathrm{~h})$ en Licence qu'en Doctorat. Si c'est une coïncidence, on peut conserver la progression 180/120/80 crédits pour L/M/D en considérant que pour le Doctorat qui est un niveau spécifique, 1 crédit $=3600 / 80=45 \mathrm{~h}$.

\subsubsection{Dégénérescence de l'état fondamental des niveaux universitaires : « bac + » comme critère d'admission au supérieur}

Lors des délibérations à l'occasion du baccalauréat sénégalais par exemple, on distingue quatre catégories de candidatas à l'issue des épreuves finales :

$1^{\mathrm{e}}$ catégorie : Candidats Admis d'Office au Premier Groupe avec Mention (CAOM).

$2^{\mathrm{e}}$ catégorie : Candidats Admis d'Office au Premier Groupe avec Mention Passable (CAOMP).

$3^{\mathrm{e}}$ catégorie : Candidats Admis au Premier Groupe après Repêchage (CAPGR).

$4^{\mathrm{e}}$ catégorie : Candidats Admis à l'issu des épreuves du Deuxième Groupe (CADG).

Supposons qu'un groupe d'étudiants issu de ces quatre catégories de bacheliers soient orientés à l'Université pour s'inscrire pour la première fois en Licence 1. On conçoit clairement qu'il se pose un réel problème de niveau pour les étudiants occupant le niveau L1. Si l'enseignant fait le focus sur les étudiants $\mathrm{CADG}$ en perdant du temps à expliquer des détails qui portent 
généralement sur des lacunes qu’ils trainent, les étudiants CAOM pourraient être frustrés d'une part par la présence d'étudiants que leurs font perdre du temps et, d'autre part, par une série de rappels qui ne leur apprend rien de nouveau. On comprend aisément que le niveau fondamental de la Licence 1 est quatre fois dégénéré (fig.8). Une levée de dégénérescence est nécessaire pour un enseignement efficace en Licence 1. Pour une comparaison avec les systèmes atomiques, nous considérons le cas de l'effet Zeeman normal sur le niveau $2 \mathrm{p}$ de l'atome d'hydrogène soumis à un champ magnétique uniforme. Ce qui donne trois sous-niveaux déterminés par les valeurs possibles du nombre quantique magnétique orbital $m_{l}=-1,0,+1$. L'éclatement du niveau fondamental de la Licence 1 est dû à ce que nous appelons «Effet Prérequis ». Les prérequis sont les connaissances de base que doit posséder un apprenant pour comprendre le cours qui va se dérouler. Ainsi, par un simple contrôle de prérequis, l'enseignant arrive à distinguer les apprenants qui sont disposés à suivre normalement son cours et ceux qui éprouveront des difficultés. Il devient évident, que l'enseignant en salle de Licence 1 fournira plus d'efforts pour permettre aux étudiants CADG d'accéder en Licence 2 qu'il n'en fournira pour les étudiants CAOM. En général, les étudiants issus de la catégorie CADG n'ont pas le niveau pour suivre correctement les enseignements en Licence 1. Les lacunes qu'ils traînent au sortir de la classe de Terminales sont notées par nombre d'enseignants qui déroulent des modules en Licence 1. Dans le cas des filières scientifiques, nous éprouverons d'énormes difficultés à enseigner en Licence 1 des modules tels que l'électrostatique et la magnétostatique. Une bonne assimilation de ces deux modules requière un background solide en Mathématiques au sortir de la classe de terminale scientifique. Malheureusement, les Mathématiques constituent la bête noire des nouveaux bacheliers CADG et CAPGR orientés en Licence 1 MPCI. Il s'ensuit que l'orientation des nouveaux bacheliers en Licence 1 devrait être assujettie à une condition sine quoi none : bac + admission d'office au premier tour (un bon candidat peut trébucher et rater la mention au bac). Ainsi, tous les bacheliers des $3^{\mathrm{e}}$ [candidats admis au Premier Tour après Repêchage (CAPGR)] et $4^{\mathrm{e}}$ catégories [candidats admis à l'issu des épreuves du Deuxième Tour (CADG)] devraient être orientés dans les filières d'enseignements professionnels. Des formations professionnelles de deux années dans les domaines de l'agriculture, de l'élevage, de la pèche, de la santé, des finances, de l'environnement, des génies électrique, civil, informatique, chimique, mécanique, etc., seraient très adoptées pour les bacheliers CAPGR et CADG. L'État économiserait ainsi des milliards annuellement à travers les bourses qui seraient allouées aux étudiants CAPGR et $\mathrm{CADG}$ qui risque de faire de la «figuration » à chaque année universitaire. 


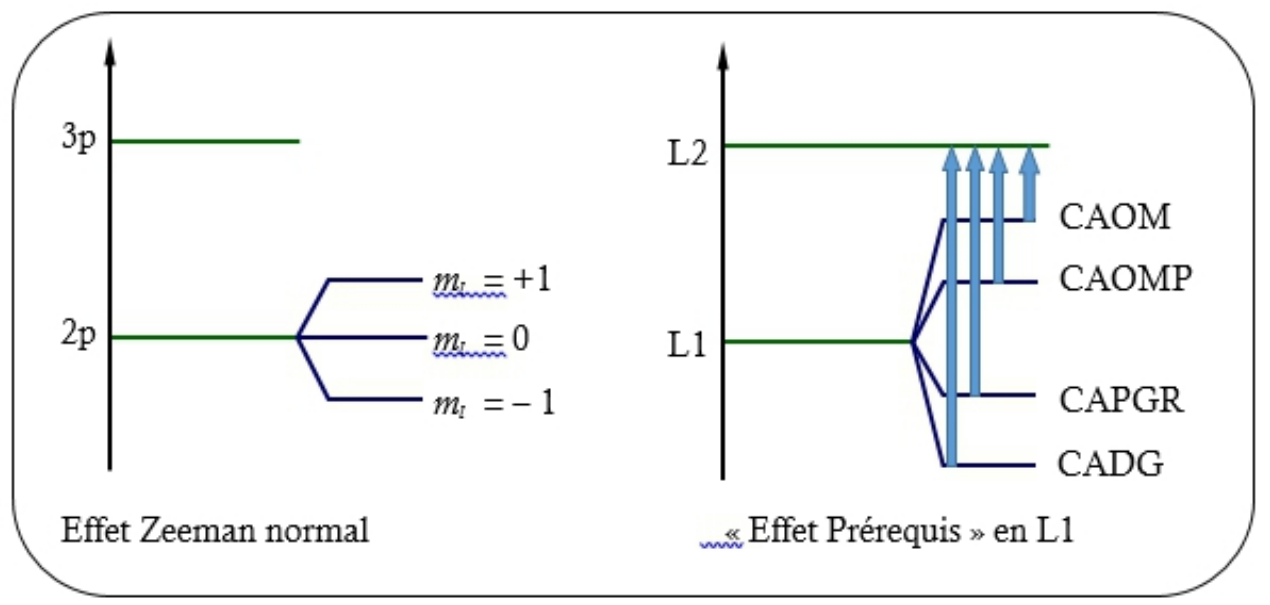

Fig.8. Analogie de l'effet Zeeman normal en pédagogie : la Licence 1 éclate en quatre composantes : l'une d'elles (composante CADG) doit disparaitre pour ne garder que trois sous-niveaux

\subsubsection{Mise en pratique d'une pédagogie discriminatoire pour un enseignement efficace}

Comme nous l'avons précisé précédemment, l'inscription de bacheliers CAPGR et CADG en Licence 1 rend la tâche difficile à l'enseignant qui aura la lourde tâche de gérer une classe à plusieurs vitesses. Pour un système idéal constitué de deux niveaux (fig.9), l'enseignement devient plus facile puisque tous les étudiants CAOM et CAOMP ont en général les prérequis nécessaires pour suivre les enseignements à dérouler. Mais un étudiant ayant la mention Bien ne peut pas être comparé à un étudiant ayant la mention Passable. Par conséquent, pour le même niveau de Licence 1 , on devrait mettre en ouvre une pédagogie discriminatoire pour un enseignement efficace (fournir tout juste l'effort nécessaire pour atteindre les objectifs escomptés) en L1. Ceci reviendrait à discriminer positivement les enseignements en L1 pour permettre aux étudiants CAOM de donner le maximum d'eux-mêmes. Ainsi, tous les étudiants CAOM et CAOMP recevront des enseignements en $\mathrm{CM}$. L'enseignant proposera des séries d'exercices « corsés » adaptés aux capacités cognitives des étudiants CAOM tout en restant dans les limites des objectifs à atteindre en L1. Ce qui fournirait d'excellents étudiants CAOM en L2 pour avoir reçu une formation renforcée en L1 du fait de leur niveau spécifique d'instruction. Les étudiants CAOM peuvent facilement être rendus autonomes dans la construction de leurs savoirs. Ce qui n'est pas le cas des étudiants CAOMP qui traînent souvent des lacunes. La différence de niveau entre les étudiants CAOM et CAOMP (fig.9) rend difficile la tâche à l'enseignant qui trouve être confronté à gérer une classe à double vitesse. L'idéal serait alors de dédoubler la classe : une L1 CAOM et une autre L1 CAOMP, chaque niveau étant confié à un enseignant 
tout en respectant le ratio apprenants/enseignant pour un niveau donné. Dans le cas général, la pédagogie discriminatoire relève de la pédagogie active. L'apprenant est donc au centre des activités d'enseignements apprentissages, l'enseignant jouant le rôle de facilitateur. Pour intégrer l'élémentaire et le moyen secondaire, on considère le fait qu'un apprenant faible ou moyen assimilera plus difficilement les concepts développés dans un cours qu'un apprenant intelligent voire doué ou surdoué. Distinguons alors deux groupes d'apprenants : les apprenants faibles et moyens et les apprenants bons et excellents.

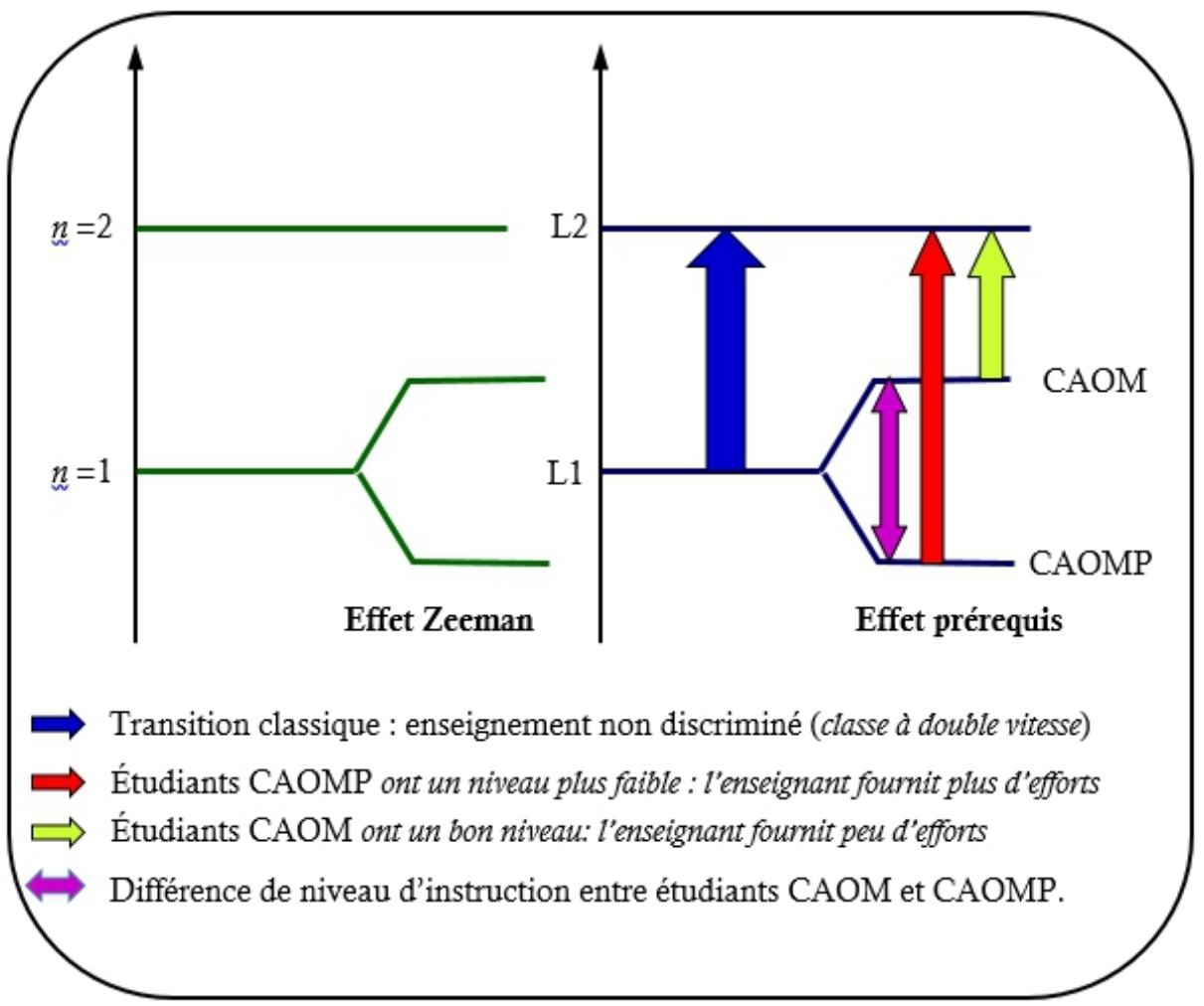

Fig.9. Effet Zeeman et Effet Prérequis : le niveau fondamental de la Licence 1 est deux fois dégénéré.

La pratique de la pédagogie discriminatoire repose essentiellement sur trois points :

1. Éclatement de la classe pédagogie en deux groupes ;

2. Mise en pratique d'un enseignement discriminé ;

3. Mise en pratique de mécanismes d'évaluations discriminés.

Le contenu de chacun de ces trois points est décliné dans ce qui suit. 


\section{- Éclatement de la classe pédagogie en deux groupes}

Le problème qui se pose est de savoir comment faire pour détecter dans une classe, un apprenant de niveau d'instruction faible ou excellent? C'est là où le contrôle de prérequis trouve toute sa pertinence. En début d'année, l'administration de l'établissement scolaire ou universitaire organise des séances d'évaluation diagnostique consistant en des tests de contrôle de prérequis sur l'ensemble des connaissances antérieurement enseignées et pour toutes les disciplines. Par exemple, pour des apprenants en classe de CM2, le contrôle de prérequis portera sur les matières enseignées en classe de CM1. Pour des apprenants en classe de Seconde, le contrôle portera sur les matières enseignées en classe de Troisième et ainsi de suite. Le critère de sélection des apprenants faibles, moyens, bons et très bons ou excellents se fera sur la base des notes obtenues et assorties d'une mention. Tous les apprenants qui auront une note inférieure à 12/20 (note correspondant à la mention Assez Bien) seront considérés comme ayant un niveau d'instruction moyen. Les apprenants de bons niveaux d'instruction seront ceux-là qui auront obtenus des notes supérieures à 12/20. Toutefois, entre ces deux catégories d'apprenants (moyens et bons) on pourrait trouver des apprenants faibles (notes inférieures à $10 / 20)$ voire très faibles $(5 / 20)$ ainsi que de très bons (notes supérieure à 14/20) voire d'excellents apprenants (notes supérieure à16/20). Dans le cas de l'enseignement élémentaire, les moyennes sont majorées à 10/10. Il pourra être considéré comme moyen un apprenant ayant une note inférieure à 5/10 et comme bon celui qui aurait obtenu une note supérieure à $5 / 10$. On voit dès lors à travers cette catégorisation, que la tâche de l'enseignent s'avère très délicate : comme faire un enseignement de qualité dans une classe constituée d'apprenants de niveaux d'instruction très variés (très faibles, faibles, moyens, bons et très bons)? Tout le génie de l'enseignant est ici invoqué. Ce qui justifie la pertinence de la mise en pratique d'une pédagogie discriminatoire pour un enseignement de qualité en classe.

\section{- Mise en pratique d'un enseignement discriminé}

Posons que les apprenants sont répartis dans les classes CAFM (Classe Apprenants Faibles et Moyens) et CABE (Classe Apprenants Bons et Excellents). Un apprenant de niveau très faible sera considéré comme faible. De même, un apprenant de niveau très bon ou excellent sera considéré comme bon. Au niveau élémentaire, la CAFM pourrait être une classe de CM2A et la CABE une classe de CM2B. On peut aussi avoir à titre d'exemples les classes $3^{\text {ème }} \mathrm{A}$ et $3^{\text {ème }} \mathrm{B}$ au moyen, les classes $2^{\text {nd }} \mathrm{A}$ et $2^{\text {nd }} \mathrm{B}$ au secondaire, Licence 1 A et Licence 1 B à l'Université.

La discrimination de l'enseignement pour une même classe, consiste à adopter la démarche pédagogique la plus adaptée au niveau d'instruction des apprenants CAFM ou CABE. Quatre démarches pédagogiques sont 
généralement mises en pratique lors d'une séance enseignement apprentissage:

- Démarche analogique qui consiste à transposer à un nouveau contexte, un traitement ou une solution déjà connue ;

- Démarche déductive consistant à aller du général au particulier. En d'autres termes, elle consiste à exposer ce qui doit être appris en commençant par un énoncé d'ordre général pour finir par des exercices d'application, donc par des cas particuliers ;

- Démarche inductive qui est l'inverse de la démarche déductive : on part du particulier, pour aller au général et revenir ensuite au particulier ;

- Démarche dialectique consistant en une approche contradictoire permettant de traiter les données par leur confrontation simultanée.

Les approches analogique et inductive seraient très adaptées au niveau d'instruction des apprenants CAFM. En revanches, les approches déductive et dialectique seraient plutôt réservées aux apprenants CABE. Á titre d'exemple illustratif, on peut citer le cas des sciences expérimentales. La démarche inductive s'appuie sur l'expérimentation faisant appel aux organes des sens (toucher, observer, sentir, entendre) tandis que la démarche déductive s'appuie sur une approche purement théorique sollicitant fortement l'esprit. Le concret de la démarche inductive fait place à l'abstrait (l'imagination) qui sous-tend l'approche déductive. La démarche inductive est préconisée en Chimie et en Physique par exemple qui sont généralement des sciences expérimentales (cette démarche n'est pas adaptée dans le cadre de la physique théorique ni de la chimie quantique). Quant à la démarche déductive, elle demeure le domaine de prédilection des Mathématiques qui sont par essence, des sciences hypothético-déductives. Une telle démarche serait plutôt privilégiée pour les apprenants CABE. De plus, l'enseignement doit avoir une bonne maîtrise de la didactique de la discipline enseignée. En effet, la ppédagogie réfère à l'apprenant alors que la didactique réfère à l'enseignement, c.-à-d. à la chose enseignée : le concept, le contenu. De plus, la pédagogie est généraliste (mise en pratique de la pédagogie active appliquée à toute discipline) alors que la didactique est spécifique. C'est ainsi qu'on parle de la didactique de la Physique ou de la didactique des Mathématiques. En outre, la pédagogie se focalise sur les relations entre l'enseignant et l'apprenant et sur les relations entre les apprenants eux-mêmes. Par contre, la didactique fait l'hypothèse fondamentale que la spécificité des contenus est déterminante dans l'appropriation des connaissances. Les différences entre pédagogie et didactique sont résumées ci-dessous (Sakho, 2018). 

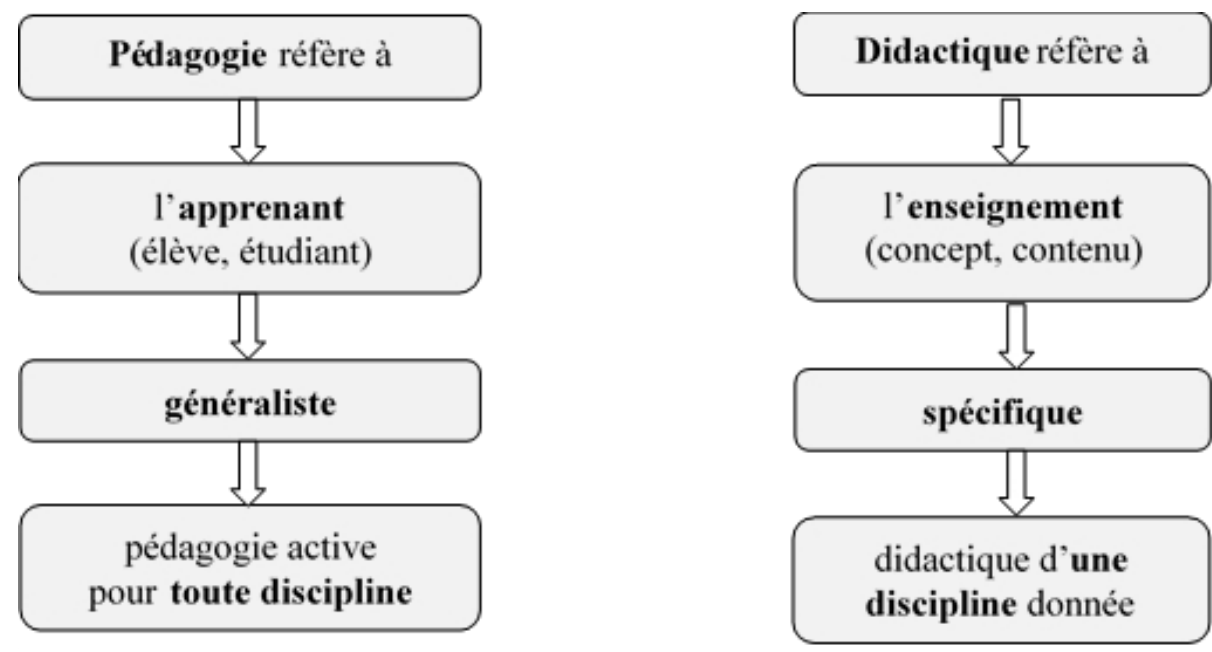

Ainsi, la mise en pratique de la pédagogie discriminatoire exige de l'enseignant de faire preuve de pédagogie et de didactique. Par ailleurs, avec l'introduction des TIC (Technologies de l'Information et de la Communication) dans les systèmes éducatifs, les enseignants désireux de mettre en pratique la pédagogie discriminatoire doivent avoir une bonne maîtrise de l'outil informatique et doivent pouvoir naviguer aisément sur internet. En effet, l'évolution des méthodes d'enseignement vers la pédagogie active a entraîné naturellement une évolution du travail du pédagogue vers un rôle de guide et de conseiller. L'introduction dans l'enseignement des TIC participe activement à l'évolution des méthodes pédagogiques puisqu'une bonne partie de l'enseignement des contenus est prise en charge par les TIC. C'est ainsi que le rôle de l'enseignant va se déplacer progressivement du contenu à enseigner vers l'enseigné (apprenant), avec un suivi qui sera de plus en plus personnalisé et une participation accrue des apprenants. L'enseignant devient ainsi un «facilitateur », une personne ressource vers qui se tourner l'apprenant en cas de besoin (il peut donc se passer de l'enseignant s'il est de la catégorie $\mathrm{CABE}$ ). Le rôle de l'enseignant s'apparente dès lors à celui d'un tuteur (qu'il soit physique : enseignement en présentiel ou à distance (tutorat à distance) ou à celui d'un coach (à l'image l'entraîneur d'une équipe) dans ce qu'on appelle le coaching pédagogique. Le coach pédagogique se distingue du coach classique (entraineur): il assume un rôle à vocation pédagogique. Il peut-être un enseignant, un professionnel ou un pair (par exemple un étudiant formé pour cela). Le coach pédagogique est souvent en situation d'encadrement puis, en situation d'évaluateur du travail fourni par l'apprenant (le tuteur n'évalue pas). Le coaching pédagogique est pratiqué dans des activités telles que par exemple l'apprentissage par projet. Le tutorat est plus réservé à la pédagogie active au niveau universitaire. Dans le système du tutorat, l'enseignant se charge de la partie théorique et le tuteur se réserve la 
mise en application pratique des enseignements. Dans l'impossibilité d'éclater le niveau en deux classes, deux groupes de TD (et de TP éventuellement) pourraient être créés : le groupe 1 constitué par les apprenants faibles et moyens et le groupe 2 par les apprenants de bons niveaux. L'enseignant donnerait alors un support de cours (format papier et/ou numérique) aux apprenants du groupe 1 et confiera le reste de son travail à un tuteur. Ce qui lui permettrait alors de consacrer plus de temps aux apprenants du groupe 2 . Toutefois, il est nécessaire une harmonisation des actions interactives enseignant-tuteur à travers des réunions de concertation et de coordination qui peuvent se faire à distance (tutorat à distance). Le tutorat à distance peut se faire suivant deux voies :

1. soit en autonomie dans l'institution (université) où se déroule le tutorat à travers un contact direct tuteur-apprenant;

2. soit suivant une interactivité à distance à travers un projet du type FOAD (Formation Ouvertes A Distance).

Le projet FOAD propose une solution globale incluant les outils informatiques qui vont faciliter la formation par exemple par visio-conférence ; messagerie ; forum de discussion ; plateforme d'échanges via internet, etc. Un tel projet est très adapté aux apprenants CABE. Toutefois, on doit garder à l'esprit que l'approche FOAD bien que révolutionnaire est difficilement applicables dans des pays connaissant des coupures intempestives de courant. De plus, la mise en ouvre correcte de la FOAD nécessite une très bonne connectivité internet (accès facile au WIFI par exemple dans tous l'espace universitaire sachant que les apprenants - étudiants - doivent pouvoir participer au forum de discussion à tout moment, qu'ils soient dans le campus pédagogique ou dans le campus social). Dans des pays où les établissements souffrent du manque d'infrastructures en informatique, la mise en pratique de la pédagogie discriminatoire consisterait à distribuer aux apprenants $\mathrm{CABE}$ des supports de cours sous formats papiers (résumés de cours plus exercices avec solutions abrégées). L'enseignant passera alors plus de temps à des applications concrètes des concepts introduits dans le cours sous forme de TD, de TP et de sorties pédagogiques. Ce qui rendrait les apprenants CABE très autonomes dans la construction de leurs savoirs.

\section{- Mise en pratique de mécanismes d'évaluations discriminés}

Tout enseignement met en interaction l'enseignant, l'apprenant et la chose enseignée: le programme (élémentaire, moyen-secondaire) ou le syllabus (université). L'outil pédagogique dont dispose l'enseignant pour vérifier le degré d'assimilation des connaissances transmises aux apprenants est : l'évaluation. Ainsi, à la fin du déroulement du cours, l'enseignant proposé des séries de TD aux apprenants. Dans le cas des disciplines à caractère expérimental comme les Sciences Physiques et les Sciences de la 
Vie et de la Terre, des séances de TP sont organisées pour une bonne appropriation des contenus pouvant faire l'objet d'études expérimentales. Ce qui permet le transfert de compétences de savoir-faire expérimental. Dans la perspective de la mise en œuvre de la pédagogie discriminatoire, il n'est pas nécessaire de différencier la conduite des TP. En effet, tous les TP sont articulés généralement entour des objectifs spécifiques suivants :

1. énumérer le matériel expérimental ;

2. faire la liste des produits à utiliser ;

3. décliner le protocole expérimental ;

4. exécuter la manipulation ;

5. interpréter les résultats expérimentaux ;

6. tirer la loi (éventuellement) ;

7. tirer une conclusion

Ces objectifs spécifiques de savoir-faire expérimental ne sont pas discriminables. Les apprenants CAFM et CABE doivent mettre la main à la patte de la même manière (les $\mathrm{CABE}$ pourraient être plus rapides dans le déroulement des TP que les CAFM). Pour ce qui concerne les séances de TD, les exercices proposés sont articulés autour des objectifs d'enseignement: chaque objectif spécifique décliné dans la fiche du cours magistral doit faire l'objet d'une évaluation pour vérifier est ce que l'apprenant est capable de..... Le choix des exercices doit nécessairement suivre la taxonomie de Bloom : on part du simple au complexe du point de vue du niveau de difficulté. Généralement, divers outils sont proposés selon trois niveaux de difficultés.

- le premier niveau consiste en une simple restitution des connaissances du cours. Les phrases à trou, les QCM, les Vrai ou Faux sont très adaptés dans ces genres d'opérations ;

- le second niveau correspondant à une aptitude à reproduire un exercice traiter en classe. Des exercices d'applications directes du cours sont adaptés à ce deuxième niveau avec la possibilité de formuler des QRC ;

- enfin, le troisième niveau de difficulté est lié à l'aptitude à transférer des connaissances du cours à un problème similaire de celui étudié en classe ou à un problème radicalement différent.

Au dernier niveau de difficulté correspond des exercices variés à travers lesquels plusieurs compétences de savoir et de savoir-faire théorique ou pratique sont évaluées. Souvent, un coup de pouce est nécessaire pour des exercices considérés comme difficiles par l'enseignant. Le coup de pouce consiste à un ensemble de conseils indiqués en marge des exercices jugés difficiles (repéré par un signe) pour aider l'apprenant dans sa résolution. De plus, les éléments fondamentaux d'une fiche de TD sont les suivants : 
1. Liste des objectifs spécifiques ;

2. Classification des objectifs spécifiques en termes d'objectifs de savoir, de savoir-faire théorique et de savoir-faire expérimental ;

3. Discrimination des objectifs spécifiques selon le niveau de difficulté des exercices à proposer.

Discriminer les objectifs spécifiques revient à les catégoriser pour voir ceux qui peuvent être évalués à l'aide de :

- d'exercices de type Vrai ou Faux, QCM et QRC ;

- d'exercices variés à caractère non expérimental ;

- d'exercices variés à caractère expérimental ;

- d'exercices variés nécessitant un coup de pouce.

Dans le cadre de la pédagogie discriminatoire, il n'est pas pertinent de donner des exercices du type Vrai ou Faux, QCM et QRC aux apprenants CABE. Leur bon niveau d'instruction leur permet de se passer de ces exercices autocorrectifs consistant à une simple restitution des connaissances du cours. Ainsi, les exercices relatifs au troisième niveau de difficulté seront proposés préférentiellement aux apprenants $\mathrm{CABE}$ en réservant les exercices de difficulté moindre aux apprenants CAFM. Notons que les TD et TP pourraient être assistés par ordinateur pour les apprenants CABE. Sur la figure 10, nous avons illustré la mise en pratique de la pédagogie discriminatoire pour les apprenants $\mathrm{CABE}$ dans une salle informatique équipée (un ordinateur par apprenant, réseau WIF permanent). L'enseignant occupe le centre de la salle. Les séances enseignement-apprentissage se faisant de façon interactive (tous les ordinateurs étant interconnectés). De plus, chacun des acteurs (enseignant et apprenants) dispose d'un microphone pour dialoguer oralement.

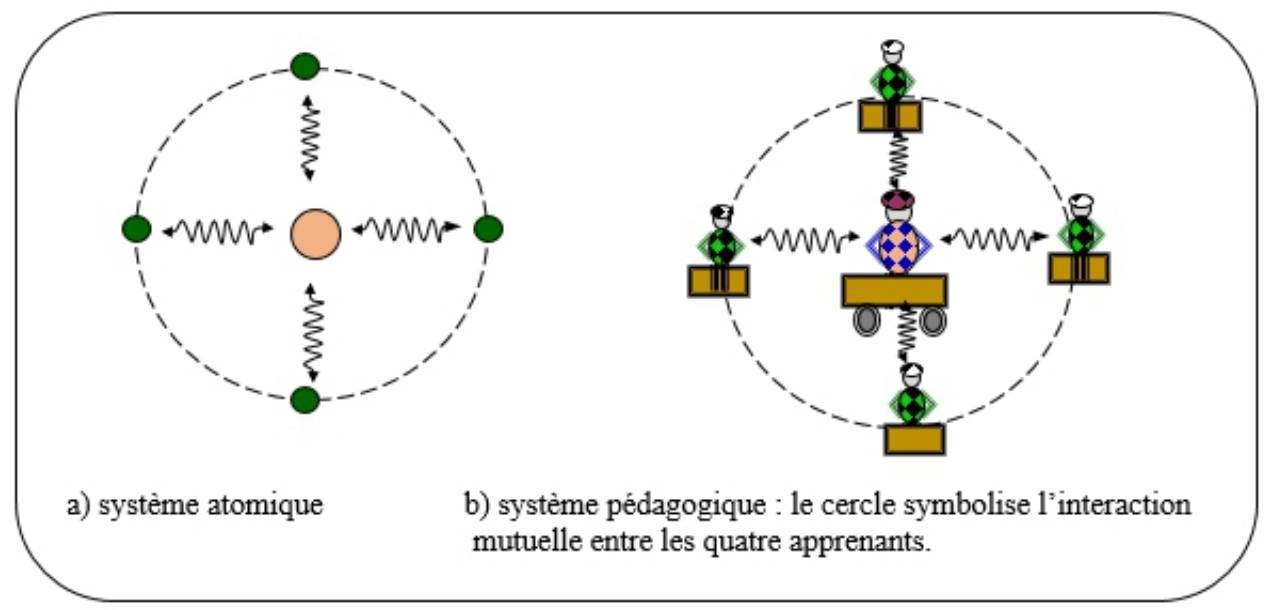

Fig.10. Mise en pratique d'une pédagogie discriminatoire pour de bons apprenants dans le cadre du projet FOAD 


\section{Conclusion}

Dans ce travail, nous avons montré que plusieurs éléments dénombrables en pédagogie sont au nombre de 7 conformément aux diverses activités de l'homme qui se déroulent en 7 jours répétés au cours de la vie. Ce qui nous a permis d'énoncer un principe appelé « principe 7 » de la pédagogie. De plus, nous avons montré que tous les niveaux d'enseignement apprentissages sont dégénérés et qu'ils sont constitués de sous-niveaux. La prise en compte de l' «Effet prérequis » permet de lever cette dégénérescence. L'analyse de l'architecture du système LMD a permis de montrer que le niveau spécifique $\mathrm{D}$ devrait correspondre à 80 crédits, un crédit valant $45 \mathrm{~h}$. De plus, l'analyse du niveau de Licence 1 a permis de montrer que l'orientation des bacheliers à l'université devrait tenir compte du critère fondamental d'admission dès le premier Groupe. La nécessité de mettre en œuvre une pédagogie discriminatoire en L1 a été aussi discutée et justifiée par les niveaux d'instruction différents des étudiants s'inscrivant pour la retire fois en Licence 1. Un résultat important a été aussi trouvé dans ce travail : nombre de bacheliers devraient plutôt être orientés dans les filières professionnelles pour leur permettre de s'insérer dans le monde du travail après deux années d'études post-baccalauréat. Ce qui diminuerait les déperditions académiques observées dans nos universités. En perspectives, il serait intéressant de confronter le principe 7 de la pédagogie au nombre de salles de classe par cycle ainsi qu'au nombre d'apprenants total pour un établissement scolaire donné. De plus, par analogie avec les processus de génération de rayonnement laser, il serait possible de montrer que le cerveau humain (constitué entre autre d'atomes) est «quantifié » et d'analyser les traitements des informations par le cerveau comme des processus de transitions « cervelloniques » entre niveaux cérébraux quantifiés. Les cervellons seraient des particules biologiques chargées d'assurer la transmission de l'information à travers tout le cerveau. Dans cette perspective, on considère que les niveaux cérébraux de traitement et de restitution des informations sont de trois types : un niveau fondamental de réception de l'information (exemple question posée par un enseignant à un apprenant) ; un niveau excité de traitement de l'information (niveau de réflexion chez l'apprenant) et un niveau intermédiaire de restitution de l'information traitée (réponse à la question posée). Un processus de relaxation (élimination de toutes les mauvaises réponses) se produirait entre le niveau cérébral de traitement de l'information et le niveau cérébral d'émission de l'information traitée. Ce qui ouvre implicitement des pistes de recherches en vue d'applications concrètes de la physique quantique en pédagogie. Une telle étude est en projet. 


\section{References:}

1. Baldé I, Bop, S \& Faye A, Sénégal: Classes pléthoriques dans les établissements scolaires publics - Quand l'école dessert les apprenants : www.fr.allafrica.com/stories/201711060652.html, 2017.

2. Bouaouina. K. L'enfer de la surcharge des classes. www.letemps.com.tn/article/104802/l'enfer-de-la-surcharge-desclasses, 2017.

3. Buisson, $\mathrm{F}$ « Nouveau dictionnaire de pédagogie», Paris, Hachette, p. 532 (1911).

4. Ceides, Processus de Bologne, vers un espace européen de l'enseignement supérieur, Ministère de la Culture, de l'Enseignement Supérieur et de la Recherche, Luxemburg, cedies@mcesr.État.lu, 2004.

5. Centre de Ressources et d'ingénierie documentaires (CRID). Introduction au processus de Bologne, www.ciep.fr/sites/default/files/atoms/.../focus-introduction-auprocessus-de-bologne.p, 2016

6. Choquette D, 9 bienfaits de la marche https://www.journaldemontreal.com/2017/04/07/9-bienfaits-de-lamarche, 2017.

7. Despres B, Les équivalences pour le baccalauréat et les formations professionnalisantes, http://www.jeunes-a-l-etranger.com/etudierangleterre-diplomes/, 2016

8. Grossetette $\mathrm{CH}$., Relativité restreinte et structure atomique de la matière, Éditions Ellipses Marketing, Paris (1985).

9. Karin. S-L, La mise en cuvre du Processus de Bologne en France et en Allemagne, HAL https://tel.archives-ouvertes.fr/,2009.

10. Lanza, R., Biocentrism, How Life and Consciousness are the Keys to Understand The True Nature of The Universe. Amazon Books, USA, (2010).

11. MENFP : Ministère de l'Education Nationale et de la Formation Professionnelle, https://www.doc developpement-durable. org /file/.../normes_constructions_scolaire.pd.., 2010.

12. Milbert, B., Physique quantique, biologie et médecine, www.docplayer.fr/17076068-Physique-quantique-biologie-etmedecine.html/, 2012.

13. Napargalè. M. Éducation nationale / Redressement de l'école : Voici les propositions des acteurs du système. https://news.abidjan.net/h/427062.html, 2012.

14. Nénard C. Les bienfaits du vélo après quelques semaines. https://www.vivonsvelo.fr/bienfaits-velo-apres-quelques-semaines23/, 2017. 
15. Revue trimestrielle d'éducation comparée (Paris, UNESCO : Bureau international d'éducation), vol. XXIII, $\mathrm{n}^{\circ} 1-2$, mars-juin 1993, p. 379406.

16. Sakho, I., L'Histoire de l'atome : de l'antiquité à nos jours. Journée de jumelage des classes de Seconde $S_{D}$ et de Première, jeudi 29 mai 2008, Lycée de Bambey, Sénégal.

17. Sakho. I., Histoire de l'atome, Éditions Publibook, Paris (2011).

18. Sakho. I., Expliquer la structuration des niveaux d'enseignementapprentissage de l'élémentaire à l'Université par la quantification des niveaux d'énergie des systèmes atomiques, Université Assane Seck de Ziguinchor, 31 mai 2013, Sénégal.

19. Sakho. I., Evolution des modèles atomiques (1902-1927) : à la découverte des nombres quantiques principal, orbital et de spin. Université Alioune Diop de Bambey, 22 décembre 2016b, Sénégal.

20. Sakho. I., Présentation du LMD et des débouchés de la filière Physique - Chimie, Journée d'intégration de l'amicale des étudiants des départements de physique et Chimie de l'UFR Sciences et Technologies de l'Université Assane Seck de Ziguinchor, 24 février 2018, Sénégal.

21. Sakho. I., Cours de pédagogie universitaire pour doctorants, Université Assane Seck de Ziguinchor, Sénégal (2018).

22. UNESCO, Ratio

élèves/enseignant http://uis.unesco.org/fr/node/334769, 2018.

23. Wikipédia. https://fr.wikipedia.org/wiki/Adolphe_Ferrière, 2018. 